\title{
SPECTRAL SYNTHESIS FOR BANACH ALGEBRAS, II
}

\author{
J. F. Feinstein and D. W. B. Somerset \\ Joel.Feinstein@nottingham.ac.uk \\ ds@maths.abdn.ac.uk
}

\begin{abstract}
This paper continues the study of spectral synthesis and the topologies $\tau_{\infty}$ and $\tau_{r}$ on the ideal space of a Banach algebra, concentrating particularly on the class of Haagerup tensor products of $\mathrm{C}^{*}$-algebras. For this class, it is shown that spectral synthesis is equivalent to the Hausdorffness of $\tau_{\infty}$. Under a weak extra condition, spectral synthesis is shown to be equivalent to the Hausdorffness of $\tau_{r}$.
\end{abstract}

1991 Maths Subject Classification 46H10, 46K50

\section{Introduction}

The notion of spectral synthesis is well-established for commutative Banach algebras and for $L^{1}$-group algebras. In [23] the second author introduced a definition of spectral synthesis for a general unital Banach algebra. The motivation behind this was the discovery that a unital commutative Banach algebra $A$ has spectral synthesis if and only if the topology $\tau_{\infty}$, introduced by Beckhoff [3] on the set of closed ideals of $A$, is Hausdorff [23; 2.6]. The definition of spectral synthesis introduced in [23] was modelled on the properties of $\mathrm{C}^{*}$ algebras, because $\tau_{\infty}$ was also known to be Hausdorff for this class [3]. The hope was that spectral synthesis might be equivalent to the Hausdorffness of $\tau_{\infty}$ for non-commutative Banach algebras. It was shown in [23] that this was so for separable, unital PI-Banach algebras, and that in general the Hausdorffness of $\tau_{\infty}$ implies a weak form of spectral synthesis. Conversely a strong form of spectral synthesis implies that the topology $\tau_{\infty}$ is $T_{1}$.

Because $\tau_{\infty}$ is seldom Hausdorff, the second author introduced another topology $\tau_{r}$ on the set $\operatorname{Id}(A)$ of closed two-sided ideals of a Banach algebra $A$ [24]. This topology is always compact, like $\tau_{\infty}$, and it is Hausdorff whenever $\tau_{\infty}$ is Hausdorff [24; 3.1.1], and often even when $\tau_{\infty}$ is not. This is the case, for instance, with TAF-algebras [24], and with the algebra $C^{1}[0,1][10]$. On the other hand it was shown in [10] that for uniform algebras, $\tau_{r}$ is Hausdorff if and only if $\tau_{\infty}$ is Hausdorff. In [24] it was shown that if there is a compact Hausdorff topology on a subspace of $\operatorname{Id}(A)$, which is related to the quotient norms in a useful way, then that topology necessarily coincides with the restriction of $\tau_{r}$. Thus for uniform algebras without spectral synthesis, such as the disc algebra, there is no useful compact Hausdorff topology on the space of closed ideals.

This paper continues the study of the relationship between spectral synthesis and the Hausdorffness of $\tau_{\infty}$ and $\tau_{r}$. A slightly modified definition of spectral synthesis is introduced, for various reasons, and several of the results of [23] are extended to the non-unital situation. In particular it is shown that the problem of proving that the Hausdorffness of 
$\tau_{\infty}$ implies spectral synthesis is harder than one might imagine - one would first have to prove that there are no non-trivial algebraically simple Banach algebras.

In the second part of the paper, we turn our attention to the class of Banach algebras obtained by taking the Haagerup tensor product of two $\mathrm{C}^{*}$-algebras. There are various reasons for looking at this class. One is that spectral synthesis has already been studied for these algebras, and it is easy to find examples where synthesis holds, and others where it fails. A second reason is that the presence of the $\mathrm{C}^{*}$-algebras makes the class reasonably tractable, and a considerable amount is already known about the ideal structure [1], [2]. A third reason is that the Banach algebras in this class are in general neither Banach *-algebras, nor operator algebras, so it might be hoped that the class is reasonably typical of semi-simple Banach algebras as a whole. We are able to show that spectral synthesis is equivalent to the Hausdorffness of $\tau_{\infty}$ for this class.

In the final part of the paper we work with the same class of Banach algebras, this time considering the topology $\tau_{r}$. We show that if the $\mathrm{C}^{*}$-algebras are unital and have the property that every closed prime ideal is maximal then their Haagerup tensor product has spectral synthesis if and only if the topology $\tau_{r}$ is Hausdorff. One novel feature of our approach is the use of the theory of continuous lattices.

We now give the definitions of the various topologies on $\operatorname{Id}(A)$, starting with the lower topology $\tau_{w}$. Let $A$ be a Banach algebra. A subbase for $\tau_{w}$ on $\operatorname{Id}(A)$ is given by the sets $\{I \in I d(A): I \nsupseteq J\}$ as $J$ varies through the elements of $I d(A)$. Thus the restriction of $\tau_{w}$ to the set of closed prime ideals is simply the hull-kernel topology. Next we define $\tau_{\infty}$. For each $k \in \mathbf{N}$, let $S_{k}=S_{k}(A)$ denote the set of seminorms ('seminorm' means 'algebra seminorm' in this paper) $\rho$ on $A$ satisfying $\rho(a) \leq k\|a\|$ for all $a \in A$. Then $S_{k}$ is a compact, Hausdorff space [3]. We say that $\rho \geq \sigma$, for $\rho, \sigma \in S_{k}$, if $\rho(a) \leq \sigma(a)$ for all $a \in A$. The point of this upside-down definition is that if $\rho \geq \sigma$ then $\operatorname{ker} \rho \supseteq \operatorname{ker} \sigma$. Clearly if $\rho, \sigma \in S_{k}$ the seminorm $\rho \wedge \sigma$ defined by $(\rho \wedge \sigma)(a)=\max \{\rho(a), \sigma(a)\}$ is the greatest seminorm less than both $\rho$ and $\sigma$ in the order structure. Thus $S_{k}$ is a lattice. The topology $\tau_{\infty}$ is defined on $\operatorname{Id}(A)$ as follows [3]: for each $k$ let $\kappa_{k}: S_{k} \rightarrow I d(A)$ be the map $\kappa_{k}(\rho)=\operatorname{ker} \rho$, and let $\tau_{k}$ be the quotient topology of $\kappa_{k}$ on $\operatorname{Id}(A)$. Then $\tau_{\infty}=\bigcap_{k} \tau_{k}$. Clearly each $\tau_{k}$ is compact, so $\tau_{\infty}$ is compact. It is a useful fact that for $I \in I d(A), I d(A / I)$ is $\tau_{\infty}-\tau_{\infty}$ homeomorphic to the subset $\{J \in \operatorname{Id}(A): J \supseteq I\}$ of $\operatorname{Id}(A)$ [3; Prop. 5].

Next we define the topology $\tau_{r}$, which is the join of two weaker topologies. The first is easily defined: $\tau_{u}$ is the weakest topology on $\operatorname{Id}(A)$ for which all the norm functions $I \mapsto\|a+I\|(a \in A, I \in I d(A))$ are upper semi-continuous. The other topology $\tau_{n}$ can be described in various different ways, but none is particularly easy to work with. A net $\left(I_{\alpha}\right)$ in $\operatorname{Id}(A)$ is said to have the normality property with respect to $I \in I d(A)$ if $a \notin I$ implies that liminf $\left\|a+I_{\alpha}\right\|>0$. Let $\tau_{n}$ be the topology whose closed sets $N$ have the property that if $\left(I_{\alpha}\right)$ is a net in $N$ with the normality property relative to $I \in I d(A)$ then $I \in N$. It follows that if $\left(I_{\alpha}\right)$ is a net in $\operatorname{Id}(A)$ having the normality property relative to $I \in I d(A)$ then $I_{\alpha} \rightarrow I\left(\tau_{n}\right)$. Any topology for which convergent nets have the normality property with respect to each of their limits (such a topology is said to have the normality property) is necessarily stronger than $\tau_{n}$, but $\tau_{n}$ itself need not have the normality property. Indeed the following is true. Let $\tau_{r}$ be the topology on $\operatorname{Id}(A)$ generated by $\tau_{u}$ and $\tau_{n}$. Then $\tau_{r}$ is always compact $[24 ; 2.3]$, and $\tau_{r}$ is Hausdorff if and only if $\tau_{n}$ has the normality property 
$[24 ; 2.12]$. The topology $\tau_{n}$ is always stronger than $\tau_{w}$, and so $\tau_{w}$ and $\tau_{n}$ coincide on a given subset of $\operatorname{Id}(A)$ if $\tau_{w}$ has the normality property on this subset.

Finally, we introduce an auxiliary topology, $\tau_{a}$. This is the topology generated by $\tau_{u}$ and $\tau_{1}$. Clearly we have $\tau_{u} \subseteq \tau_{a}$ and $\tau_{\infty} \subseteq \tau_{a}$. By [24;2.6], $\tau_{n} \subseteq \tau_{\infty}$ and so we also have $\tau_{r} \subseteq \tau_{a}$. We shall need this topology in the proof of Theorem 1.6.

The following simple lemma is taken from $[10 ; 0.1]$.

Lemma 0.1 Let $A$ be a Banach algebra. Let $\left(I_{\alpha}\right)$ be a net in $\operatorname{Id}(A)$, either decreasing or increasing, and correspondingly either set $I=\bigcap I_{\alpha}$ or $I=\overline{\bigcup I_{\alpha}}$. Then $I_{\alpha} \rightarrow I\left(\tau_{r}\right)$.

\section{The foundations revisited}

In this section we re-examine the foundations for the work on spectral synthesis. We introduce a slightly more general definition of spectral synthesis and consider some of its elementary consequences. This change of definition obliges us to survey the results of [23] to see how they are affected. Since we wish subsequently to work with group algebras, we also take the opportunity to liberate the theory from the requirement that the Banach algebra should be unital. This is sometimes more tricky than one anticipates. We consider some of the relations between spectral synthesis, weak spectral synthesis, $\tau_{\infty}$, and $\tau_{r}$ for commutative Banach algebras, PI-Banach algebras, and algebraically simple Banach algebras, and we also look at the stability properties of spectral synthesis on passage to ideals, quotients, and extensions.

Old definition of spectral synthesis In [23], a unital Banach algebra $A$ was said to have spectral synthesis if it has the following four properties: (i) $\operatorname{Prim}(A)$ is locally compact (i.e. every point has a neighbourhood base of compact sets), (ii) every closed subset of $\operatorname{Prim}(A)$ is a Baire space (i.e. the intersection of countably many dense open sets is dense), (iii) $\tau_{w}$ has the normality property on $\operatorname{Prim}(A)$, (iv) $\operatorname{Id}(A)$ is isomorphic to the lattice of open subsets of $\operatorname{Prim}(A)$, under the correspondence $I \leftrightarrow\{P \in \operatorname{Prim}(A): P \nsupseteq I\}$.

Here $\operatorname{Prim}(A)$ is the space of primitive ideals of $A$ (i.e. kernels of algebraically irreducible representations with the hull-kernel topology). If the word $\operatorname{Prim}(A)$ is replaced throughout by $\operatorname{Prime}(A)$ (the set of proper closed prime ideals of $A$ with the hull-kernel topology) then $A$ is said to have weak spectral synthesis, and if the word $\operatorname{Prim}(A)$ is replaced throughout by $\operatorname{Max}(A)$ (the set of closed, maximal modular ideals of $A$ with the hull-kernel topology) then $A$ is said to have strong spectral synthesis.

Unfortunately, this old definition of spectral synthesis is slightly too restrictive for the algebras that we wish to consider. In these algebras, and also in Banach *-algebras, it is natural to consider an ideal as 'primitive' if it is the kernel of a topologically irreducible *-representation on a Hilbert space, and it is not evident that such an ideal is primitive in the usual algebraic sense. We shall therefore need to relax the requirements for spectral synthesis. Note, however, that we do not change the definitions for weak and strong spectral synthesis, except for dropping the requirement of an identity element. 
There is also a second reason for wanting to modify the definition of spectral synthesis. Recall that a non-empty closed subset of a topological space is irreducible if it is not the union of two proper closed subsets. The closure of a point is a typical example of an irreducible closed set, and a topological space is said to be sober if every irreducible closed set is the closure of a point. For instance, every Hausdorff space is sober. An infinite set with the cofinite topology is not sober, because it is an irreducible closed subset of itself, but is not the closure of any of its points.

Sobriety of a space is closely connected with the Baire property. For example, a locally compact, sober space is a Baire space [12; p.84]. Indeed if $X$ is a second countable $T_{0}$-space such that the sobrification $X^{s}$ (see below) is locally compact, then $X$ is sober if and only if every closed subspace of $X$ is a Baire space [12; V.5.27(ii)]. This, of course, is axiom (ii) above, which was required for some of the arguments in [23]. Thus axiom (ii) would be redundant if $\operatorname{Prim}(A)$ were always sober. What we do, therefore, to obtain slightly more generality, is to replace $\operatorname{Prim}(A)$ by its sobrification, as follows.

Every $T_{0}$-space $X$ has a unique sobrification $X^{s}$, which may be defined in the following way. Let $X^{s}$ be the topological space whose points are the irreducible closed sets of $X$, and whose non-empty open sets have the form $\left\{A \in X^{s}: A \cap U \neq \emptyset\right\}$, where $U$ varies through the non-empty open subsets of $X$. Then $X^{s}$ is a sober space. The map $i: X \rightarrow X^{s}$, $i(x)=\{x\}^{-}$, embeds $X$ in $X^{s}$. Evidently $X$ and $X^{s}$ have isomorphic lattices of open sets, and $i(X)=X^{s}$ if and only if $X$ is sober.

For an algebra $R$ over $\mathbf{C}$, let $\operatorname{Idl}(R)$ denote the set of all two-sided ideals of $R$. An ideal $I \in \operatorname{Idl}(R)$ is said to be semisimple if it is an intersection of primitive ideals, and to be strongly semisimple if it is an intersection of maximal modular ideals. If $R$ is a Banach algebra then semisimple and strongly semisimple ideals are, of course, automatically closed.

Lemma 1.1 Let $R$ be an algebra over C. The sobrification $\operatorname{Prim}^{s}(R)$ of $\operatorname{Prim}(R)$ is (homeomorphic to) the set of semisimple, prime ideals of $R$, with the hull-kernel topology. The sobrification $\operatorname{Max}^{s}(R)$ of $\operatorname{Max}(R)$ is (homeomorphic to) the set of strongly semisimple, prime ideals of $R$, with the hull-kernel topology.

Proof. A simple argument shows that for a semisimple ideal $P$, the hull-kernel closed set $X=\{Q \in \operatorname{Prim}(R): Q \supseteq P\}$ is irreducible if and only if $P$ is prime. Thus there is a one-to-one correspondence between $\operatorname{Prim}^{s}(R)$ and the set of semisimple prime ideals, and it is straightforward to confirm that the map is a homeomorphism.

An analogous argument deals with $\operatorname{Max}(R)$. Q.E.D.

For example, if $A$ is the disc algebra then $\operatorname{Prim}(A)=\operatorname{Max}(A)$ is an irreducible closed subset of itself in the hull-kernel topology, but is not the closure of any of its points. In fact $\operatorname{Prim}^{s}(A)=\operatorname{Max}(A) \cup\{\{0\}\}$. On the other hand, since every Hausdorff space is sober, $\operatorname{Prim}^{s}(A)=\operatorname{Max}(A)$ when $A$ is a completely regular, commutative Banach algebra. If $A$ is a $C^{*}$-algebra then every closed ideal of $A$ is semisimple, so $\operatorname{Prim}^{s}(A)=\operatorname{Prime}(A)$. The famous open problem of whether every closed, prime ideal of a (non-separable) $\mathrm{C}^{*}$-algebra $A$ is primitive is thus precisely the question whether $\operatorname{Prim}(A)$ is a sober space for an arbitrary $\mathrm{C}^{*}$-algebra. 
We are now ready to introduce the new definition of spectral synthesis.

Definition 1.2 A Banach algebra $A$, unital or otherwise, has spectral synthesis if it has the following properties:

$(\mathrm{i})^{\prime}, \operatorname{Prim}^{s}(A)$ (the space of semisimple, prime ideals with the hull-kernel topology) is locally compact,

(ii) ${ }^{\prime} \tau_{w}$ has the normality property on $\operatorname{Prim}(A)$,

(iii) $)^{\prime} \operatorname{Id}(A)$ is isomorphic to the lattice of open subsets of $\operatorname{Prim}(A)$, under the correspondence $I \leftrightarrow\{P \in \operatorname{Prim}(A): P \nsupseteq I\}$. Equivalently, every proper, closed ideal of $A$ is semisimple.

A few remarks on this new definition are in order.

Remarks (a) It can be shown [12; V.5.10] that if $X$ is a $T_{0}$-space and $Y$ is locally compact with $X \subseteq Y \subseteq X^{s}$ then $X^{s}$ is locally compact. Thus if $\operatorname{Prim}(A)$ is locally compact, then (since $\operatorname{Prim}(A)$ is a $T_{0}$-space) $\operatorname{Prim}^{s}(A)$ is also locally compact. Every Banach algebra, therefore, which had spectral synthesis under the old definition still has it under the new definition. As a matter of fact, the authors do not know of a unital Banach algebra which has spectral synthesis under the new definition but not under the old.

(b) $\operatorname{Prim}^{s}(A)$ is always sober, so if it is also locally compact then every closed subspace of $\operatorname{Prim}^{s}(A)$ is a Baire space [12; V.5.27(ii)]. Thus we do not need any axiom corresponding to (ii) in the old definition of spectral synthesis.

(c) Axiom (ii)' is, of course, axiom (iii) from the old definition of spectral synthesis. It is easy to show that $\tau_{w}$ has the normality property on $\operatorname{Prim}(A)$ if and only if it has the normality property on $\operatorname{Prim}^{s}(A)$.

(d) It was shown in $[23 ; 1.1]$ that if $A$ has weak spectral synthesis then $\tau_{w}$ has the normality property on $I d(A)$. The proof works perfectly well in the non-unital case too. Hence it follows from Proposition 1.10 (below) that if $A$ has spectral synthesis (or strong spectral synthesis) then $\tau_{w}$ has the normality property on $\operatorname{Id}(A)$. This implies that $\tau_{w}$ and $\tau_{n}$ coincide on $\operatorname{Id}(A)[24 ;$ p.373 and p.375].

(e) Axiom (iii)' is, of course, axiom (iv) from the old definition of spectral synthesis. Recall that $\operatorname{Prim}(A)$ and $\operatorname{Prim}^{s}(A)$ have isomorphic lattices of open sets.

The next thing to do is to check that the new definition coincides with the standard one for commutative Banach algebras. Recall that a (possibly non-unital) commutative Banach algebra $A$ has spectral synthesis (usual definition) if the map $I \mapsto\{P \in \operatorname{Prim}(A): P \supseteq I\}$ sets up a 1-1 correspondence between closed ideals of $A$ and Gelfand closed subsets of $\operatorname{Prim}(A)$. This is equivalent to requiring that the hull-kernel and Gelfand topologies coincide on $\operatorname{Prim}(A)$, and that every closed ideal of $A$ is semisimple.

Let $A$ be a commutative Banach algebra. Then every primitive ideal of $A$ is maximal and modular, indeed the kernel of a character [18;4.2.19], but if $A$ is non-unital then $A$ might have maximal proper ideals which are not primitive. Here is an instructive example from [4; Example 2].

Example 1.3 Let $A=C[0,1]$ be the commutative $\mathrm{C}^{*}$-algebra of continuous functions on the interval $[0,1]$ with the supremum norm. Let $z \in A$ be the identity map given by 
$z(t)=t(t \in[0,1])$. Define a new multiplication $\diamond$ on $A$ by $f \diamond g=f z g$, and let $B$ be the resulting Banach algebra. Then $\operatorname{Id}(B)=\operatorname{Id}(A)$, and the character space of $B$ is the set $\left\{t \omega_{t}: t \in(0,1]\right\}$ where $\omega_{t}$ is the point evaluation at $t$. The ideal ker $\omega_{0}$ is a maximal ideal of $B$, but not a primitive ideal, and $B / \operatorname{ker} \omega_{0}$ is isomorphic to the complex numbers with the zero multiplication.

The next lemma (for which we have not been able to find a reference) shows that this example is typical.

Lemma 1.4 Let $A$ be a commutative Banach algebra and let $M$ be a maximal proper ideal of $A$. Then $A / M$ is isomorphic to $\mathbf{C}$, either with the usual multiplication or with the zero multiplication.

Proof. Set $B=A / M$. Let $b \in B \backslash\{0\}$. Then $B b$ is an ideal of $B$, so either $B b=\{0\}$ or $B b=B$. In the first case, $\{\lambda b: \lambda \in \mathbf{C}\}$ is a non-zero ideal of $B$, so $\{\lambda b: \lambda \in \mathbf{C}\}=B$. Hence $B$ is one-dimensional with zero multiplication. In the second case, there exists $u \in B$ such that $u b=b$. Since $B b=B$, for any $c \in B$ there exists $d \in B$ such that $d b=c$, and then $u c=u d b=d b=c$. Thus $u$ is the identity for $B$. Hence $M$ is a maximal modular ideal of $A$, so $M$ is closed and $B=\{\lambda u: \lambda \in \mathbf{C}\}$, see [8; $§ 16$ Theorem 5]. Q.E.D.

If $M$ above is not modular then $M$ need not be closed. For example, let $A$ be an infinitedimensional Banach space with the zero multiplication, and let $M$ be a dense hyperplane. Then $A$ is a commutative Banach algebra, and $M$ is a maximal ideal of $A$ which is not closed.

If $A$ is a commutative Banach algebra and $M$ is a maximal modular ideal of $A$ then, for $a \in A$, we shall identify the coset $a+M$ with the value that the character corresponding to $M$ takes at $a$. One inconvenience with non-unital commutative Banach algebras is that the process of evaluating at a character and taking the modulus sometimes gives a number strictly less than the quotient norm for the corresponding maximal modular ideal. This happens in Example 1.3, for instance. To get round this problem, we use the following lemma.

Lemma 1.5 Let $A$ be a commutative Banach algebra. Suppose that $\left(M_{\alpha}\right)$ is a net in $\operatorname{Prim}(A)$ and that $a \in A$ with $\lim _{\alpha} a+M_{\alpha}=0$. Then $\lim _{\alpha}\left\|a^{2}+M_{\alpha}\right\|=0$.

Proof. For any character $\phi$ on $A, a^{2}-\phi(a) a \in \operatorname{ker} \phi$. Hence

$$
\left\|a^{2}+\operatorname{ker} \phi\right\| \leq\left\|a^{2}-\left(a^{2}-\phi(a) a\right)\right\|=\|\phi(a) a\|=|\phi(a)|\|a\| .
$$

Thus $\lim \sup _{\alpha}\left\|a^{2}+M_{\alpha}\right\| \leq \lim \sup _{\alpha}\left|a+M_{\alpha}\right|\|a\|=0$. Q.E.D.

For a Banach algebra $A$, let $\mathcal{M}^{\prime}$ be the space of maximal modular ideals of codimension one. The next result was proved in [3; Proposition 11] under the additional requirement that $A$ has a bounded approximate identity (see also Maths Reviews 97f:46073, where it is observed that one only need assume that $A^{2}=A$ ). Part of [3; Proposition 11] was that the set $\mathcal{M}^{\prime} \cup\{A\}$ is $\tau_{\infty}$-closed if $A$ has a bounded approximate identity. In Example 1.3, 
however, $\operatorname{ker} \omega_{0}$ is in the $\tau_{\infty}$-closure of $\mathcal{M}^{\prime}$, so that particular part of [3; Proposition 11] cannot be extended to the general case.

Theorem 1.6 Let $A$ be a Banach algebra. Then the restrictions of the topologies $\tau_{n}, \tau_{r}$, and $\tau_{\infty}$ all coincide on the Gelfand space $\mathcal{M}^{\prime}$ of $A$ with the Gelfand topology.

Proof. The set $\mathcal{M}^{\prime}$ is $\tau_{w}$-closed in $\operatorname{Prim}(A)$ by a result of Kaplansky's, see [24; 5.1]. Set $K=\bigcap\left\{M: M \in \mathcal{M}^{\prime}\right\}$. Then $A / K$ is commutative, and $\mathcal{M}^{\prime}$ is homeomorphic, in all four topologies under consideration, to the Gelfand space of $A / K$ [3; Proposition 5], [24; 2.9]. Thus we may assume at the outset that $A$ is commutative. Under this assumption we have $\mathcal{M}^{\prime}=\operatorname{Prim}(A)$, so to complete the proof we need to show that the topologies under consideration agree on $\operatorname{Prim}(A)$.

Let $X_{1}$ be the set of closed ideals of $A$ of codimension not greater than one. Then $X_{1}$ is a $\tau_{n}$-closed subset of $\operatorname{Id}(A)[24 ; 5.1]$. By [24; 4.3(ii)] the topologies $\tau_{n}$ and $\tau_{a}$ coincide on the set of minimal elements of $X_{1}$, which is $X_{1} \backslash\{A\}$ (recall that $\tau_{a}$ is the topology generated by $\tau_{u}$ and $\tau_{1}$ ). However we know that $\tau_{n} \subseteq \tau_{\infty} \subseteq \tau_{a}$ and also $\tau_{n} \subseteq \tau_{r} \subseteq \tau_{a}$. It follows that all four of these topologies coincide on $X_{1} \backslash\{A\}$, and hence that $\tau_{n}, \tau_{r}$ and $\tau_{\infty}$ coincide on the subset $\operatorname{Prim}(A) \subseteq X_{1} \backslash\{A\}$.

Suppose that $\left(P_{\alpha}\right)$ is a net in $\operatorname{Prim}(A)$ converging to $\operatorname{P} \in \operatorname{Prim}(A)$ in the Gelfand topology. For each $\alpha$ let $c_{\alpha}$ be the seminorm in $S_{1}(A)$ given by $c_{\alpha}(a)=\left|a+P_{\alpha}\right|(a \in$ $A)$. Then $c_{\alpha} \rightarrow c$ where $c \in S_{1}(A)$ is the seminorm $c(a)=|a+P|(a \in A)$. Hence $P_{\alpha}=\operatorname{ker} c_{\alpha} \rightarrow \operatorname{ker} c=P$ in the $\tau_{\infty}$ topology. Thus the Gelfand topology on Prim $(A)$ is stronger than the relative $\tau_{\infty}$ topology.

Conversely, let $Y$ be a Gelfand-open subset of $\operatorname{Prim}(A)$ with compact closure (in the Gelfand topology). We shall show that $Z=X_{1} \backslash Y$ is $\tau_{n}$-closed in $\operatorname{Id}(A)$. This will show that $Y$, and hence every Gelfand-open subset of $\operatorname{Prim}(A)$, is $\tau_{n}$-open in $\operatorname{Prim}(A)$. It follows that the various topologies coincide on $\operatorname{Prim}(A)$.

Let $\left(I_{\alpha}\right)$ be a net in $Z$ and let $I \in I d(A) \backslash Z$. We have to show, by [24; 2.5], that there exists $a \in A \backslash I$ such that $\lim \inf \left\|a+I_{\alpha}\right\|=0$. If $I \notin X_{1}$ then this follows at once from $[24 ; 2.5]$ and the fact that $X_{1}$ itself is $\tau_{n}$-closed in $\operatorname{Id}(A)$. So suppose that $I \in Y$. If $\left(I_{\alpha}\right)$ is eventually in $X_{1} \backslash \operatorname{Prim}(A)$ then choose any $a \in A \backslash I$. Then $a^{2} \notin I$, but $a^{2} \in I_{\alpha}$ eventually by Lemma 1.4. Hence $\lim _{\alpha}\left\|a^{2}+I_{\alpha}\right\|=0$. The other possibility is that $\left(I_{\alpha}\right)$ is frequently in $\operatorname{Prim}(A)$. The local compactness of the Gelfand topology on $\operatorname{Prim}(A)$ implies that $\left(I_{\alpha}\right)$ has a subnet $\left(I_{\beta}\right)$ in $\operatorname{Prim}(A)$ such that either $\left(I_{\beta}\right)$ goes to infinity (i.e. is eventually outside every Gelfand compact subset of Prim $(A))$ or for which there exists $J \in \operatorname{Prim}(A)$ with $I_{\beta} \rightarrow J$ in the Gelfand topology. In the first case choose any $a \notin I$; in the second case choose $a \in J \backslash I$. Then in either case $a \notin I$ but $a+I_{\beta} \rightarrow 0$. Hence $\lim _{\beta}\left\|a^{2}+I_{\beta}\right\|=0$ by Lemma 1.5 , but $a^{2} \notin I$, since $I \in \operatorname{Prim}(A)$. Q.E.D.

Corollary 1.7 Let $A$ be a commutative Banach algebra. Then $A$ has spectral synthesis (in the sense of this paper) if and only if $A$ has spectral synthesis in the usual sense.

Proof. Suppose that $A$ has spectral synthesis in the sense of this paper. Then, since $\tau_{w}$ has the normality property on $\operatorname{Prim}(A), \tau_{w}$ and $\tau_{n}$ coincide on $\operatorname{Prim}(A)$. Thus the hull-kernel topology $\left(\tau_{w}\right)$ and the Gelfand topology coincide on $\operatorname{Prim}(A)$ by Theorem 
1.6. Condition (iii) ${ }^{\prime}$, on the other hand, implies that every closed ideal of $A$ is semisimple. Hence $A$ has spectral synthesis in the usual sense.

Conversely, suppose that $A$ has spectral synthesis in the usual sense. Then certainly condition (iii) ${ }^{\prime}$ holds. Furthermore, since $\operatorname{Prim}(A)$ is $\tau_{w}$-Hausdorff it is sober, so $\operatorname{Prim}(A)=\operatorname{Prim}^{s}(A)$. Thus condition (i) ${ }^{\prime}$ holds. Finally condition (ii) ${ }^{\prime}$ follows from the fact that for any $a \in A$ and $P \in \operatorname{Prim}(A),\|a+P\| \geq|a+P|$, and the function $P \mapsto|a+P|$ is $\tau_{w}$-continuous on $\operatorname{Prim}(A)$ since $A$ is completely regular. Thus $A$ has spectral synthesis in the sense of this paper. Q.E.D.

The next result was established for unital, commutative Banach algebras in $[23 ; 2.6]$. We follow exactly the same method of proof, but making use of Theorem 1.6. Recall the theorem of Rickart's [20] that if $A$ is a completely regular, semisimple, commutative Banach algebra then every norm $\|\cdot\|^{\prime}$ on $A$ is spectral, that is, for all $a \in A,\|a\|^{\prime} \geq$ $\sup \{|a+M|: M \in \operatorname{Max}(A)\}$. This implies that $\{0\}$ is $\tau_{\infty}$-closed in $\operatorname{Id}(A)$. Recall also the theorem of Bohnenblust and Karlin [7], see [25; 12.7], that if $A$ is a commutative Banach algebra and $a \in A$ then the spectral radius of $a$ is the infimum of $\|a\|^{\prime}$ over all possible norms $\|\cdot\|^{\prime}$ equivalent to the original norm, and bounded by it. Thus if $a$ is a non-zero quasinilpotent element, there is a sequence $\left(\|\cdot\|_{n}\right)$ of norms in $S_{1}(A)$ such that $\|a\|_{n} \rightarrow 0$. Considering a cluster point of such a sequence we see that $\{0\}$ is not $\tau_{\infty}$-closed in $\operatorname{Id}(A)$. It follows that a necessary condition for $\{0\}$ to be $\tau_{\infty}$-closed in $\operatorname{Id}(A)$ is that $A$ should be semisimple.

Theorem 1.8 Let $A$ be a commutative Banach algebra. Then $A$ has spectral synthesis if and only if the topology $\tau_{\infty}$ is Hausdorff on $\operatorname{Id}(A)$.

Proof. Suppose that $\tau_{\infty}$ is Hausdorff on $\operatorname{Id}(A)$. Then $\tau_{\infty}$ is Hausdorff on $\operatorname{Id}(A / I)$ for all $I \in I d(A)$ [3; Prop. 5], so $A / I$ must be semisimple, for all $I \in I d(A)$ by the theorem of Bohnenblust and Karlin just mentioned. Thus condition (iii)' holds. Furthermore [23; Theorem 2.5] shows that $\tau_{w}$ has the normality property on $\operatorname{Id}(A)$, hence on $\operatorname{Prim}(A)$, so condition (ii)' holds. It also follows that $\tau_{w}$ and $\tau_{n}$ are equal, so Theorem 1.6 shows that the Gelfand and hull-kernel topologies coincide on $\operatorname{Prim}(A)$. Hence condition (i)' holds, and $A$ has spectral synthesis.

The proof of the converse is identical to the corresponding part of the proof of [23; $2.6]$; there are no difficulties in passing to the non-unital case. Q.E.D.

In general a commutative Banach algebra can have $\tau_{r}$ Hausdorff and yet fail to have spectral synthesis. Various examples are given in [24; Section 3]. For the class of uniform algebras, however, it was shown in $[10 ; 1.2]$ that $\tau_{r}$ cannot be Hausdorff unless spectral synthesis holds. Our next theorem is a more general version of that result, valid also for non-unital Banach algebras.

Definition Let $A$ be a Banach function algebra, and let $\Gamma(A)$ denote the Shilov boundary of $A$. Recall that a Gelfand compact subset $X$ of $\operatorname{Max}(A)$ is a Helson set if $\left.A\right|_{X}=C(X)$ 
(where $C(X)$ is the algebra of continuous complex functions on $X$ ). Letting $I$ be the closed ideal consisting of elements of $A$ which vanish on $X$, the least constant $K$ such that

$$
K \sup \{|f(x)|: x \in X\} \geq\|f+I\| \text { for all } f \in A
$$

is called the Helson constant of $X$. We say that a Banach function algebra $A$ has the Helson property (with constant $k$ ) if there is a constant $k$ such that whenever $U$ is a non-empty Gelfand open subset of $\Gamma(A)$ there is an increasing net $\left(F_{\alpha}\right)_{\alpha}$ of Helson sets of constant bounded by $k$ contained in $U$ such that $\bigcup_{\alpha} F_{\alpha}$ is Gelfand dense in $U$.

For example, if $A$ is a uniform algebra then the collection of finite p-sets in the open set $U$ is an increasing net of Helson sets, with Helson constant 1, whose union is dense in $U$, see [10]. Thus uniform algebras have the Helson property with constant 1 . We shall see after Proposition 3.5 that if $A$ and $B$ are commutative $\mathrm{C}^{*}$-algebras then the Haagerup tensor product $A \otimes_{h} B$ (when viewed as a function algebra on its maximal ideal space) also has the Helson property with constant 1.

For a Banach function algebra $A$, and a Gelfand closed subset $F$ of $\operatorname{Max}(A)$, let $I(F)$ be the ideal of elements of $A$ which vanish on $F$, and let $J(F)$ be the ideal of elements of compact support vanishing in a Gelfand neighbourhood of $F$ in $\operatorname{Max}(A)$. If $F \subseteq \Gamma(A)$, let $L(F)$ be the ideal obtained as the closure of the set of elements of $A$ having compact support on $\Gamma(A)$ and vanishing in a Gelfand neighbourhood of $F$ in $\Gamma(A)$. If $\operatorname{Max}(A)=\Gamma(A)$ then of course $L(F)$ is simply the closure of $J(F)$, but this need not be true when $\operatorname{Max}(A) \neq \Gamma(A)$.

Now recall that one characterization of spectral synthesis for a Banach function algebra $A$ is that $A$ has spectral synthesis if $J(F)$ is dense in $I(F)$ for each Gelfand closed subset $F$ of $\operatorname{Max}(A)$. A related, weaker notion is that $A$ is strongly regular if $J(\{x\})$ is dense in $I(\{x\})$ for each $x \in \operatorname{Max}(A)$. If $I(\{x\})=L(\{x\})$ for each $x \in \Gamma(A)$ then $A$ is strongly regular on $\Gamma(A)$; in fact, this implies that $\Gamma(A)=\operatorname{Max}(A)$, see [11; Theorem 2] (the simple argument there is for unital Banach function algebras, but it is easily modified to cope with the non-unital case).

Theorem 1.9 Let $A$ be a Banach function algebra with the Helson property (with constant $k$ ). Then $A$ has spectral synthesis if and only if $\tau_{r}$ is Hausdorff on $\operatorname{Id}(A)$.

Proof. If $A$ has spectral synthesis then $\tau_{r}$ is Hausdorff by Theorem 1.8 and $[24 ; 3.1 .1]$. If $A$ does not have spectral synthesis, there are two possibilities. Either $\operatorname{Max}(A)=\Gamma(A)$, in which case $J(F)$ is dense in $L(F)$ for every closed subset $F$ of $\operatorname{Max}(A)$, so by assumption there is a Gelfand closed subset $X$ of $\operatorname{Max}(A)$ such that $I(X) \neq L(X)$. Otherwise $\operatorname{Max}(A) \neq \Gamma(A)$, so $A$ is not strongly regular on $\Gamma(A)$, as we remarked just above. Thus there exists $x \in \Gamma(A)$ such that $I(\{x\}) \neq L(\{x\})$. Hence in either case there is a Gelfand closed subset $X$ of $\Gamma(A)$ such that $I(X) \neq L(X)$.

Let $\left(V_{\alpha}\right)_{\alpha}$ be a net of decreasing, open neighbourhoods of $X$ in $\Gamma(A)$, each having compact complement in $\Gamma(A)$, such that $\bigcap_{\alpha} N_{\alpha}=X$ (where for each $\alpha, N_{\alpha}$ is the closure of $V_{\alpha}$ in the Gelfand topology). Then $\left(I\left(N_{\alpha}\right)\right)_{\alpha}$ is an increasing net in $\operatorname{Id}(A)$, and $I\left(N_{\alpha}\right) \subseteq$ $L(X)$, for each $\alpha$, so

$$
I:=\overline{\bigcup_{\alpha} I\left(N_{\alpha}\right)} \subseteq L(X) .
$$


For each $\alpha$, let $\left(F_{\beta(\alpha)}\right)_{\beta(\alpha)}$ be an increasing net of Helson sets in $V_{\alpha}$ of constant bounded by $k$ and whose union is dense in $V_{\alpha}$. Then $\left(I\left(F_{\beta(\alpha)}\right)\right)_{\beta(\alpha)}$ is a decreasing net in $\operatorname{Id}(A)$, and the density condition implies that $\bigcap_{\beta(\alpha)} I\left(F_{\beta(\alpha)}\right)=I\left(N_{\alpha}\right)$. Hence $I\left(F_{\beta(\alpha)}\right) \stackrel{\beta(\alpha)}{\longrightarrow} I\left(N_{\alpha}\right)$ $\left(\tau_{r}\right)$ by Lemma 0.1. But $I\left(N_{\alpha}\right) \rightarrow I\left(\tau_{r}\right)$, also by Lemma 0.1 , so if $\left(I\left(F_{\gamma}\right)\right)_{\gamma}$ denotes the 'diagonal' net, see $\left[16 ; \S 2\right.$, Theorem 4], then $I\left(F_{\gamma}\right) \rightarrow I \subseteq L(X)\left(\tau_{r}\right)$.

Suppose that $f \notin I(X)$. Then there is a Gelfand open subset $U$ of $\Gamma(A)$ meeting $X$, and an $\epsilon>0$ such that $|f(x)|>\epsilon$ for all $x \in U$. By the density condition there is, for each $\alpha$, a $\beta_{0}(\alpha)$ such that $F_{\beta_{0}(\alpha)} \cap U$ is non-empty, and hence such that $\left\|f+I\left(F_{\beta(\alpha)}\right)\right\|>\epsilon$ for all $\beta(\alpha) \geq \beta_{0}(\alpha)$. Hence the 'diagonal' net $I\left(F_{\gamma}\right) \rightarrow I(X)\left(\tau_{n}\right)$. On the other hand, if $f \in I(X)$ and $\epsilon>0$ is given then a simple topological argument shows that there exists $\alpha_{0}$ such that for $\alpha \geq \alpha_{0}, N_{\alpha} \subseteq\{x \in \Gamma(A):|f(x)|<\epsilon\}$. Thus for $\alpha \geq \alpha_{0}$,

$$
\left\|f+I\left(F_{\beta(\alpha)}\right)\right\|<k \epsilon
$$

for all $\beta(\alpha)$, by the Helson property (with constant $k$ ). Hence $I\left(F_{\gamma}\right) \rightarrow I(X)\left(\tau_{u}\right)$, using $[24 ; 2.1]$, and so

$$
I\left(F_{\gamma}\right) \rightarrow I(X)\left(\tau_{r}\right) .
$$

Since $I \subseteq L(X)$, and $L(X)$ is a strict subset of $I(X)$, we have $I \neq I(X)$, so $\tau_{r}$ is not Hausdorff. Q.E.D.

Recall that for a Banach algebra, $\tau_{r}$ is Hausdorff if and only if $\tau_{n}$ has the normality property [24; 2.12]. Thus Theorem 1.9 can be rephrased as saying that for semisimple commutative Banach algebras with the Helson property, spectral synthesis holds if and only if $\tau_{n}$ has the normality property. In this form, Theorem 1.9 is evidently closely related to Beckhoff's result [4; Proposition 3, Theorem 6] that for semisimple commutative Banach algebras with the 'distance property', spectral synthesis holds if and only if $\tau_{w}$ has the normality property.

We now need to check that the change of definition of spectral synthesis has not spoiled the results of the previous paper [23]. Recall the definitions of weak and strong spectral synthesis from [23], given at the beginning of this section. We extend these to the nonunital case simply by dropping the requirement for $A$ to be unital. Note that the Baire property, axiom (ii), is superfluous for weak spectral synthesis, since $\operatorname{Prime}(A)$ is always sober, and hence automatically a Baire space when it is locally compact.

First we observe that the change in definition of spectral synthesis allows us to remove the separability restriction from $[23 ; 1.2]$.

Proposition 1.10 Let $A$ be a Banach algebra. If $A$ has strong spectral synthesis then $A$ has spectral synthesis. If $A$ has spectral synthesis then $A$ has weak spectral synthesis.

Proof. Suppose that $A$ has strong spectral synthesis. Then every proper closed ideal of $A$ is strongly semisimple, so $\operatorname{Max}^{s}(A)=\operatorname{Prim}^{s}(A)$ by Lemma 1.1. Thus condition (i) ${ }^{\prime}$ of Definition 1.2 holds, since $\operatorname{Max}^{s}(A)$ is locally compact by Remark (a) after Definition 1.2. Condition (ii) ${ }^{\prime}$ follows by an easy modification of $[23 ; 1.1]$, and condition (iii) ${ }^{\prime}$ is trivial. Thus $A$ has spectral synthesis. 
Now suppose that $A$ has spectral synthesis. Then every closed ideal of $A$ is semisimple, so $\operatorname{Prime}(A)=\operatorname{Prim}^{s}(A)$ by Lemma 1.1. The remarks after Definition 1.2 now establish that $A$ has weak spectral synthesis. Q.E.D.

Now that weak spectral synthesis has been defined for non-unital Banach algebras, we can observe that [23; Theorem 2.9] extends to the non-unital case. The only place in the proof where the identity was used was in the appeal to [23; Corollary 2.8], but that corollary is valid for non-unital Banach algebras (simply adjoin an identity). Thus we have the following.

Theorem 1.11 Let $A$ be a Banach algebra and suppose that $\tau_{\infty}$ is Hausdorff on $\operatorname{Id}(A)$. Then $A$ has weak spectral synthesis.

We do not know in general whether weak spectral synthesis implies spectral synthesis. It was remarked in [23] that an infinite-dimensional, simple, radical Banach algebra (if there is one) would have weak spectral synthesis, but not spectral synthesis. Thus even in the commutative case it could be that weak spectral synthesis does not imply spectral synthesis.

The next result, motivated by discussion with Peter Dixon, shows that the general problem of showing that $\tau_{\infty}$ being Hausdorff on $\operatorname{Id}(A)$ implies that $A$ has spectral synthesis is more difficult than might appear. One would first have to show that there are no algebraically simple, radical Banach algebras - a famous old problem. Such an algebra would not have spectral synthesis, but would have $\tau_{\infty}$ Hausdorff, as we now show. The following lemma is certainly not new.

Lemma 1.12 Let $A$ be an algebraically simple Banach algebra of dimension greater than one, and let $x$ be a non-zero element of $A$. Set

$$
A x A=\left\{\sum_{i=1}^{n} a_{i} x b_{i}: a_{1}, \ldots, a_{n}, b_{1}, \ldots, b_{n} \in A\right\}
$$

Then $A x A=A$.

Proof. The set $A x A$ is an ideal. If $A x A=\{0\}$ for some non-zero $x$, then the ideal $I=\{x \in A: A x A=\{0\}\}$ would be non-zero. Hence $I$ would equal $A$. This would imply that $A^{3}=\{0\}$, and this would lead immediately to the existence of non-zero proper ideals. Q.E.D.

Theorem 1.13 Let $A$ be an algebraically simple Banach algebra of dimension greater than one. Then the topology $\tau_{\infty}$ is Hausdorff on $\operatorname{Id}(A)$.

Proof. The point $A$ is always $\tau_{\infty}$-closed in $\operatorname{Id}(A)$, so we need only show that $\{0\}$ is $\tau_{\infty^{-}}$ closed. To do this we shall show that for each non-zero $x \in A$ there is a constant $C>0$ 
such that $\rho(x) \geq C / k^{3}$ for all non-zero $\rho \in S_{k}$. Set $I=A x A$. Then $I=A$ by Lemma 1.12 , so $I^{2}=A$ (since $\left.A^{2} \neq 0\right)$. Thus there exist $a_{i}, b_{i}, c_{i} \in A(1 \leq i \leq n)$ such that

$$
x=\sum_{i=1}^{n} a_{i} x b_{i} x c_{i}
$$

Let $\rho \in S_{k}$. Then

$$
0<\rho(x) \leq \sum_{i=1}^{n} \rho\left(a_{i}\right) \rho(x) \rho\left(b_{i}\right) \rho(x) \rho\left(c_{i}\right) \leq \rho(x)^{2} \sum_{i=1}^{n} k^{3}\left\|a_{i}\right\|\left\|b_{i}\right\|\left\|c_{i}\right\| .
$$

Thus

$$
1 \leq \rho(x) \sum_{i=1}^{n} k^{3}\left\|a_{i}\right\|\left\|b_{i}\right\|\left\|c_{i}\right\|
$$

SO

$$
C=\left\{\sum_{i=1}^{n}\left\|a_{i}\right\|\left\|b_{i}\right\|\left\|c_{i}\right\|\right\}^{-1}
$$

is the required constant. Q.E.D.

Although we do not know in general whether weak spectral synthesis implies spectral synthesis, we shall show in a moment (Theorem 1.17) that this implication holds for semisimple PI-Banach algebras, and hence in particular for semisimple commutative Banach algebras. First we need to show that the various forms of spectral synthesis have reasonable stability properties.

Proposition 1.14 Let $A$ be a Banach algebra with weak spectral synthesis. If $I \in \operatorname{Id}(A)$ and $J \in I d(I)$ then $J \in I d(A)$.

Proof. We show that $J$ is a union of ideals of $A$.

For $a \in A$, let $I_{a}$ be the smallest closed, two-sided ideal of $A$ containing $a$. Evidently $I_{a} \subseteq I$ if $a \in I$. By condition (iv) of the definition of weak spectral synthesis, $I_{a}=\bigcap\{P \in$ $\operatorname{Prime}(A): a \in P\}$. It is clear that $I_{a} \supseteq(A a A)^{-}$. On the other hand, if $P \in \operatorname{Prime}(A)$ and $a \notin P$ then $A a A \nsubseteq P$. Thus $P \supseteq A a A \Leftrightarrow P \supseteq I_{a}$, so condition (iv) implies that $(A a A)^{-}=I_{a}$.

Note too that $\left(K^{3}\right)^{-}=K$ for all $K \in I d(A)$, since a closed prime ideal contains $\left(K^{3}\right)^{-}$if and only if it contains $K$. Hence

$$
\begin{aligned}
& I_{a}=\left(\left(I_{a}\right)^{3}\right)^{-}=\left(I_{a}(A a A)^{-} I_{a}\right)^{-}=\left(I_{a}(A a A) I_{a}\right)^{-} \\
& =\left(\left(I_{a} A\right) a\left(A I_{a}\right)\right)^{-} \subseteq\left(I_{a} a I_{a}\right)^{-} \subseteq(A a A)^{-}=I_{a} .
\end{aligned}
$$

Thus $I_{a}=\left(I_{a} a I_{a}\right)^{-}$. It follows that

$$
J \supseteq \bigcup_{a \in J}(I a I)^{-} \supseteq \bigcup_{a \in J}\left(I_{a} a I_{a}\right)^{-}
$$




$$
=\bigcup_{a \in J} I_{a} \supseteq \bigcup_{a \in J}\{a\}=J .
$$

Hence $J=\bigcup_{a \in J} I_{a}$, so $J \in I d(A)$. Q.E.D.

Corollary 1.15 Let $A$ be a Banach algebra with spectral synthesis (or weak, or strong spectral synthesis) and let $J \in \operatorname{Id}(A)$. Then $J$ and $A / J$ have spectral synthesis (or weak, or strong spectral synthesis, respectively).

Proof. First we give the proof for spectral synthesis. Conditions (i) ${ }^{\prime}$ and (iii) ${ }^{\prime}$ of Definition 1.2 follow for $A / J$ from the canonical homeomorphisms between $\operatorname{Prim}(A / J)$ and $\{P \in$ $\operatorname{Prim}(A): P \supseteq J\}$, and $I d(A / J)$ and $\{I \in I d(A): I \supseteq J\}$, respectively [21; 2.6.6]. Condition (ii)' follows from the fact that the first of these homeomorphisms preserves the quotient norms.

The spectral synthesis for $J$ is slightly less obvious. Condition (i) ${ }^{\prime}$ for $J$ follows from the homeomorphism $P \mapsto P \cap J$ between $\{P \in \operatorname{Prim}(A): P \nsupseteq J\}$ and $\operatorname{Prim}(J)$. Condition (ii) $^{\prime}$ for $J$ follows by using this homeomorphism and condition (ii)' for $A$, and noting that

$$
\|b+P\| \leq\|b+P \cap J\| \quad(b \in J, P \in \operatorname{Prim}(A)) .
$$

Condition (iii) ${ }^{\prime}$ for $J$ follows from condition (iii) ${ }^{\prime}$ for $A$ together with Proposition 1.14.

The proof for weak spectral synthesis follows in the same way. For strong spectral synthesis, the only additional feature is the need to establish condition (ii), the Baire property for closed subsets. Each closed subset of $\operatorname{Max}(A / J)$ is homeomorphic to a closed subset of $\operatorname{Max}(A)$, and hence has the Baire property by condition (ii) for $A$. Now, suppose that $M$ is a closed subset of $\operatorname{Max}(J)$, and that $\left(X_{i}\right)_{i \geq 1}$ is a countable collection of dense open subsets of $M$. Let $N$ be the closure of $M$ in $\operatorname{Max}(A)$ (regarding $\operatorname{Max}(J)$ as a subset of $\operatorname{Max}(A)$ in the usual way). Then each $X_{i}$ is dense in $N$, and open since $\operatorname{Max}(J)$ is open in $\operatorname{Max}(A)$. But $N$ has the Baire property by condition (ii) for $A$, so $\bigcap_{i=1}^{\infty} X_{i}$ is dense in $N$, and thus in $M$. It follows that $M$ has the Baire property. Q.E.D.

It would be interesting to know whether the extension property holds for spectral synthesis, that is, suppose that $A$ is a Banach algebra and that $J \in I d(A)$ with $J$ and $A / J$ both having spectral synthesis. Does $A$ have spectral synthesis? We do not know the answer even in the special cases when $A$ is commutative, or when $A$ is the unitization of $J$. The best that we have is the following.

Proposition 1.16 Let $A$ be a Banach algebra with $J \in I d(A)$, and suppose that $J$ and $A / J$ have spectral synthesis. Then every closed ideal of $A$ is semisimple.

Proof. We introduce the following temporary notation. For a Banach algebra $B$, and $I \in I d(B)$, let $h_{B}(I)=\{P \in \operatorname{Prim}(B): P \supseteq I\}$. Now let $\pi: A \rightarrow A / J$ denote the quotient map. Let $I, K \in I d(A)$ with $h_{A}(I)=h_{A}(K)$. We shall show that $I=K$. First note that the assumption on $I$ and $K$ implies that $h_{A / J}(\pi(I))=h_{A / J}(\pi(K))$ and hence that $\pi(I)=\pi(K)$, by spectral synthesis for $A / J$. On the other hand, we also have that $h_{J}(I \cap J)=h_{J}(K \cap J)$, and hence that $I \cap J=K \cap J$, by spectral synthesis for $J$. 
Now let $b \in I$. Then there exists $c \in K$ such that $\pi(b)=\pi(c)$, and hence such that $b-c \in J$. But this means (see the proof of Proposition 1.14) that

$$
\begin{gathered}
(b-c) \in(J(b-c) J)^{-} \subseteq(J b J-J c J)^{-} \\
\subseteq(J I J-J K J)^{-} \\
\subseteq(I \cap J-K \cap J)^{-}=K \cap J .
\end{gathered}
$$

Hence $b=c-(c-b) \in K+K \cap J=K$. Thus $I \subseteq K$. Similarly, $K \subseteq I$. Q.E.D.

Note that the proof of Proposition 1.16 only uses the fact that every closed ideal in $J$ and $A / J$ is semisimple. Proposition 1.16 raises the interesting question of whether a Banach algebra for which every closed ideal is semisimple automatically has spectral synthesis. We shall see that this is the case for the class of examples which we consider in the next section. In general the answer to the question is not known even for commutative Banach algebras.

We can now show that for semisimple PI-Banach algebras, weak spectral synthesis is equivalent to spectral synthesis. Let us introduce some notation. If $A$ is a Banach algebra, and $P$ is the kernel of an irreducible representation of $A$ of dimension $k$ then $P$ has codimension $k^{2}$. Let $X_{k}(k \geq 1)$ be the set of primitive ideals of $A$ of codimension less than or equal to $k^{2}$, and let $X_{0}$ be the empty set. Kaplansky showed that $X_{k}$ is a $\tau_{w^{-}}$-closed subset of $\operatorname{Prim}(A)[15 ;$ p.237].

Recall that an algebra is said to be a $P I$-algebra if there is a non-trivial polynomial identity satisfied by the elements of $A$ (for example, all $X, Y, Z \in M_{2}(\mathbf{C}$ ), the $2 \times 2$ complex matrices, satisfy $\left[[X, Y]^{2}, Z\right]=0$, where $[.,$.$] denotes the additive commutator). We shall$ say that $A$ is a PI-algebra of degree $m$ if $A$ satisfies a polynomial of degree $m$, but of no smaller degree. If $A$ is a Banach algebra satisfying a polynomial identity of degree $m$ then every primitive ideal of $A$ is maximal and every irreducible representation of $A$ has dimension less than or equal to $m / 2$, see $[9 ; 10.4 .6]$ and $[18 ; 7.1 .16]$. If $A$ is a semisimple PI-Banach algebra of degree $m$ then $A$ has an irreducible representation of degree $m / 2$.

Theorem 1.17 Let $A$ be a PI-Banach algebra with weak spectral synthesis. If $A$ is semisimple then $A$ has spectral synthesis.

Proof. It is enough to show that every proper closed prime ideal of $A$ is primitive.

Suppose first that all the primitive ideals of $A$ are of the same finite codimension $n^{2}$. Let $R$ be any proper closed ideal of $A$ of codimension not greater than $n^{2}$. Then $A / R$ is finite-dimensional, and semiprime, so $A / R$ is semisimple by Wedderburn's theorem [18; 8.1.1]. Thus $R \in \operatorname{Prim}(A)$, by the assumption on dimensions. This shows that $\operatorname{Prim}(A)$ coincides with the set of proper closed ideals of $A$ of codimension not greater than $n^{2}$. By $[24 ; 5.1]$, this set is $\tau_{n}$-closed in $I d^{\prime}(A)$ (the set of all proper, closed, two-sided ideals of $A$ ). Also, since $\tau_{w}$ has the normality property on $\operatorname{Prime}(A), \tau_{w}$ and $\tau_{n}$ coincide on $\operatorname{Prime}(A)$. Thus $\operatorname{Prim}(A)$ is $\tau_{w}$-closed in $\operatorname{Prime}(A)$. On the other hand, $\operatorname{Prim}(A)$ is $\tau_{w}$-dense in $\operatorname{Prime}(A)$ because $A$ is semisimple $[24 ; 1.1]$. Thus $\operatorname{Prim}(A)=\operatorname{Prime}(A)$. 
Now suppose that $A$ is any PI-Banach algebra of degree $2 n$, with weak spectral synthesis. Let $J_{i}=\bigcap X_{i}(0 \leq i \leq n)$ (so $J_{n}=\{0\}$, and $J_{0}=A$ ). Let $P \in \operatorname{Prime}(A)$ and let $m \in\{0, \ldots, n-1\}$ be the smallest number such that $P \nsupseteq J_{m}$. Thus $P \supseteq J_{m+1}$. Let $\pi: A \rightarrow A / J_{m+1}$ be the quotient map. Set $B=A / J, Q=\pi(P)$, and $I=\pi\left(J_{m}\right)$. Then $Q \in \operatorname{Prime}(B)$ and $Q \in \operatorname{Prim}(B)$ if and only if $P \in \operatorname{Prim}(A)$. Since $Q \nsupseteq I, Q \cap I \in$ $\operatorname{Prime}(I)$, and $Q \cap I \in \operatorname{Prim}(I)$ if and only if $Q \in \operatorname{Prim}(B)$. But $\operatorname{Prim}(I) \cong X_{m} \backslash X_{m-1}$, and all the primitive ideals of $I$ are of the same finite codimension $m^{2}$. Furthermore $I$ has weak spectral synthesis by Corollary 1.15 . Thus $\operatorname{Prime}(I)=\operatorname{Prim}(I)$ by the previous paragraph, so $Q \cap I \in \operatorname{Prim}(I)$. Hence $P \in \operatorname{Prim}(A)$, as required. Q.E.D.

Combining Theorems 1.11 and 1.17, we get the following. The unital case was established in $[23 ; 2.10]$.

Corollary 1.18 Let $A$ be a PI-Banach algebra with $\tau_{\infty}$ Hausdorff on $\operatorname{Id}(A)$. Then $A$ has spectral synthesis.

Proof. By Theorem 1.11 and Theorem 1.17 we need only show that $A$ is semisimple. But $A$ is semiprime, by Theorem 1.11, so if the Jacobson radical of $A$ were non-zero then there would be a non-zero quasi-nilpotent element in the centre of $A$ by Rowen's theorem [9; 10.7.5] (every non-zero ideal in a semiprime PI-algebra has non-zero intersection with the centre). As was observed in Remark (ii) after Proposition 2.7 of [23], such an element is all that is needed to construct a net of norms in $S_{1}(A)$ converging to a seminorm with a non-zero kernel, and this would contradict the Hausdorffness of $\tau_{\infty}$. Thus $A$ is semisimple. Q.E.D.

A partial converse to Corollary 1.18 was established in [23; 3.10]: if $A$ is a separable, unital PI-Banach algebra with spectral synthesis then $\tau_{\infty}$ is Hausdorff.

\section{Haagerup tensor products of $\mathrm{C}^{*}$-algebras}

In this section we start looking at spectral synthesis and the topology $\tau_{\infty}$ for the Haagerup tensor product $A \otimes_{h} B$ of two $C^{*}$-algebras $A$ and $B$. We show that if $A$ and $B$ are separable then every closed prime ideal of $A \otimes_{h} B$ is primitive, just as for $\mathrm{C}^{*}$-algebras. For general $A$ and $B$ we show that $A \otimes_{h} B$ has spectral synthesis if and only if $\tau_{\infty}$ is Hausdorff on $\operatorname{Id}\left(A \otimes_{h} B\right)$.

For $\mathrm{C}^{*}$-algebras $A$ and $B$, the Haagerup norm $\|\cdot\|_{h}$ is defined on an element $x$ in the algebraic tensor product $A \otimes B$ by

$$
\|x\|_{h}=\inf \left\|\sum_{i=1}^{n} a_{i} a_{i}^{*}\right\|^{1 / 2}\left\|\sum_{i=1}^{n} b_{i}^{*} b_{i}\right\|^{1 / 2},
$$

where the infimum is taken over all possible representations of $x$ as a finite sum $x=$ $\sum_{i=1}^{n} a_{i} \otimes b_{i}, a_{i} \in A, b_{i} \in A$. The completion of $A \otimes B$ in this norm is called the Haagerup 
tensor product. The norm is a Banach algebra norm; introductory information on the Banach algebra $A \otimes_{h} B$ can be found in [5], [1], and [2]. Since this is still something of a specialist area, we assemble a few useful facts. The first two are the most remarkable.

(i) Injectivity. If $A$ and $B$ are $\mathrm{C}^{*}$-algebras, and $C$ and $D$ are $\mathrm{C}^{*}$-subalgebras of $A$ and $B$ respectively, then the algebraic embedding $C \otimes D \rightarrow A \otimes_{h} B$ extends to an isometric embedding of $C \otimes_{h} D$ in $A \otimes_{h} B$ [19].

(ii) Exactness. If $A$ and $B$ are $\mathrm{C}^{*}$-algebras and $I \in I d(A), J \in I d(B)$, then there is a short exact sequence

$$
0 \rightarrow I \otimes_{h} B+A \otimes_{h} J \rightarrow A \otimes_{h} B \rightarrow A / I \otimes_{h} B / J \rightarrow 0 .
$$

(iii) If $A$ and $B$ are both infinite-dimensional $\mathrm{C}^{*}$-algebras then $A \otimes_{h} B$ is not bicontinuously isomorphic to a Banach subalgebra of the algebra of bounded operators on a Hilbert space $[6 ; 2.2]$.

(iv) The natural involution on the algebraic tensor product $A \otimes B$ of two $\mathrm{C}^{*}$-algebras $A$ and $B$ does not in general extend to an involution on $A \otimes_{h} B$, but if $A$ and $B$ are abelian then the involution does extend, and $A \otimes_{h} B$ is a Banach ${ }^{*}$-algebra.

(v) The Banach algebra $A \otimes_{h} B$ is semisimple [1; 5.16].

(vi) The Haagerup tensor product $A \otimes_{h} B$ of two abelian $\mathrm{C}^{*}$-algebras $A$ and $B$ is bicontinuously isomorphic to their projective tensor product $A \hat{\otimes} B$, see $[1 ; \mathrm{p} .112]$. Thus $A \otimes_{h} B$ is in this case a completely regular, semisimple commutative Banach ${ }^{*}$-algebra.

We shall use these various properties without further comment.

The first thing to do now is to consider the various different spaces of 'primitive' ideals of $A \otimes_{h} B$. Recall that $\operatorname{Prim}^{s}\left(A \otimes_{h} B\right)$ is the sobrification of $\operatorname{Prim}\left(A \otimes_{h} B\right)$ (the space of primitive ideals of $A \otimes_{h} B$ ) and consists of the semisimple prime ideals of $A \otimes_{h} B$. It follows from [1; 5.10, 2.6, and 5.16] that every closed prime ideal of $A \otimes_{h} B$ is semisimple. Thus $\operatorname{Prim}^{s}\left(A \otimes_{h} B\right)=\operatorname{Prime}\left(A \otimes_{h} B\right)$, the set of proper, closed, prime ideals of $A \otimes_{h} B$, and hence the notions of spectral synthesis and weak spectral synthesis coincide for the class of Haagerup tensor products of $\mathrm{C}^{*}$-algebras. Furthermore $\operatorname{Prim}^{s}\left(A \otimes_{h} B\right)$ is homeomorphic to $\operatorname{Prime}(A) \times \operatorname{Prime}(B)$, by $[1 ; 5.10]$ and $[2 ; 1.5]$, so $\operatorname{Prim}^{s}\left(A \otimes_{h} B\right)$ is always locally compact. Thus condition (i)' of spectral synthesis is always satisfied for $A \otimes_{h} B$. The same is true for condition $(\text { ii) })^{\prime}$, by $[2 ; 3.4]$. Thus the only condition that needs to be checked for spectral synthesis is (iii) ${ }^{\prime}$ - whether every closed ideal of $A \otimes_{h} B$ is semisimple.

Notice that we can therefore solve the extension problem for algebras in this class with spectral synthesis. It follows from the remark after Proposition 1.16 that if $M$ is an algebra in this class with a closed ideal $J$ such that both $J$ and $M / J$ have spectral synthesis then $M$ has spectral synthesis.

The other space of 'primitive' ideals that we have to bear in mind is $\operatorname{Prim}_{*}\left(A \otimes_{h} B\right)$, defined as follows. A representation $\pi$ of $A \otimes_{h} B$ on a Hilbert space $H$ is a $*$-representation if $\pi\left(a^{*} \otimes b^{*}\right)=\pi(a \otimes b)^{*}$ for $a \in A, b \in B$. Let $\operatorname{Prim}_{*}\left(A \otimes_{h} B\right)$ be the space of kernels of topologically irreducible $*$-representations of $A \otimes_{h} B$, with the hull-kernel topology. Elements of $\operatorname{Prim}_{*}\left(A \otimes_{h} B\right)$ are prime and semisimple [1; 5.13(ii), 5.10, 5.16], so $\operatorname{Prim}_{*}\left(A \otimes_{h} B\right) \subseteq \operatorname{Prim}^{s}\left(A \otimes_{h} B\right)$, with equality holding if $A$ and $B$ are separable [1; 
5.15]. In Corollary 2.3 we shall show that, for separable $A$ and $B$, these two spaces also coincide with $\operatorname{Prim}\left(A \otimes_{h} B\right)$. Caution is required in using results from [1] and [2] because the definition of 'primitive' used there corresponds to our definition of elements of $\operatorname{Prim}_{*}\left(A \otimes_{h} B\right)$ (in particular, the statement of $[1 ; 5.13(\mathrm{i})]$ is identical to that of our Theorem 2.2!).

Ideals of $A \otimes_{h} B$ of the form $I \otimes_{h} J(I \in I d(A), J \in I d(B))$ are called product ideals. Clearly each product ideal is generated by the simple tensors (i.e. tensors of the form $a \otimes b, a \in A, b \in B$ ) which it contains. An ideal which is generated by the product ideals which it contains is called a lower ideal, see $[1 ; 6.7]$. To define upper ideals, we use the observation of Blecher's [5] that if $A$ and $B$ are two $\mathrm{C}^{*}$-algebras then the Haagerup tensor product $A \otimes_{h} B$ is canonically isomorphic to a dense subalgebra of the minimal $\mathrm{C}^{*}$-tensor product $A \otimes_{\min } B$. A closed ideal in $A \otimes_{h} B$ of the form $J \cap A \otimes_{h} B\left(J \in I d\left(A \otimes_{\min } B\right)\right)$ is called an upper ideal $[1 ; \S 6]$. It was shown in $[1 ; 6.7]$ that an ideal in $\operatorname{Id}\left(A \otimes_{h} B\right)$ is an upper ideal if and only if it is an intersection of elements of $\operatorname{Prim}_{*}\left(A \otimes_{h} B\right)$. Each closed ideal $I$ is contained in a unique smallest upper ideal $I^{u}$, and contains a unique largest lower ideal $I_{l}$. Spectral synthesis is said to hold at $I$ if $I^{u}=I_{l}$ [1; p.140], and $A \otimes_{h} B$ was said in [1] to have spectral synthesis if every closed ideal has spectral synthesis. It is known that every product ideal has spectral synthesis; indeed the sum of a finite number of product ideals is itself a closed ideal $[1 ; 3.8]$ and has spectral synthesis $[1 ; 6.4]$. Since every primitive ideal is closed and prime, and every closed prime ideal is a sum of two product ideals $[1 ; 5.9]$, it follows that every primitive ideal has spectral synthesis. In particular, every primitive ideal of $A \otimes_{h} B$ is an upper ideal. On the other hand, since every element of $\operatorname{Prim}_{*}\left(A \otimes_{h} B\right)$ is semisimple, it follows that an ideal of $A \otimes_{h} B$ is an upper ideal if and only if it is semisimple.

Putting all this together, we have established the following.

Proposition 2.1 Let $A$ and $B$ be $C^{*}$-algebras. Then the Haagerup tensor product $A \otimes_{h} B$ has spectral synthesis in the sense of this paper if and only every closed ideal of $A \otimes_{h} B$ has spectral synthesis in the sense of [1].

Examples (i) If $A$ and $B$ are $\mathrm{C}^{*}$-algebras with $\operatorname{Id}(A)$ or $\operatorname{Id}(B)$ finite then $A \otimes_{h} B$ has spectral synthesis, by $[1 ; 5.3,6.4]$. Thus there are lots of non-commutative Banach algebras which are not $\mathrm{C}^{*}$-algebras but which have spectral synthesis, e.g. take the Haagerup tensor product of any infinite-dimensional $\mathrm{C}^{*}$-algebra with the compact operators, or with the Fermion algebra.

(ii) Let $X$ and $Y$ be countable, locally compact, Hausdorff spaces. Then $C_{0}(X) \otimes_{h}$ $C_{0}(Y)$ has spectral synthesis. To see this, we argue as follows. First note that $C_{0}(X) \otimes_{h}$ $C_{0}(Y)$ is a closed ideal in $C\left(X^{\prime}\right) \otimes_{h} C\left(Y^{\prime}\right)$ (where $X^{\prime}$ and $Y^{\prime}$ are the one-point compactifications of $X$ and $Y$ respectively), so by Corollary 1.15 we may assume that $X$ and $Y$ are compact. It now follows by transfer methods, see [13; 11.1.3], that there is a locally compact abelian group $G$ such that $C(X) \otimes_{h} C(Y)$ is bicontinuously isomorphic to a quotient of $L^{1}(G)$ by a semisimple ideal $J$. Thus $X \times Y$ is homeomorphic to the hull of $J$ in $\widehat{G}$. But every countable closed subset of $\widehat{G}$ is a set of synthesis, see [22; 7.2.5], from which it follows that $C(X) \otimes_{h} C(Y)$ has spectral synthesis. 
(iii) If $D$ is the Cantor set then the commutative Banach algebra $C(D) \otimes_{h} C(D)$ does not have spectral synthesis (because the projective tensor product $C(D) \hat{\otimes} C(D)$, with which it is isomorphic, does not have spectral synthesis, see $[13 ; 11.2 .1])$.

We now show that if $A$ and $B$ are separable $C^{*}$-algebras then every closed prime ideal of $A \otimes_{h} B$ is primitive. For a $\mathrm{C}^{*}$-algebra $A$, let $\tilde{A}$ denote the unitization of $A$ (that is, $A$ itself if $A$ is unital, and $A$ with an identity adjoined otherwise).

Theorem 2.2 Let $A$ and $B$ be $C^{*}$-algebras, and let $P \in \operatorname{Prim}(A)$ and $Q \in \operatorname{Prim}(B)$. Then $P \otimes_{h} B+A \otimes_{h} Q \in \operatorname{Prim}\left(A \otimes_{h} B\right)$.

Proof. Since $A \otimes_{h} B /\left(P \otimes_{h} B+A \otimes_{h} Q\right) \cong A / P \otimes_{h} B / Q[1 ; 2.5]$, it is enough to show that $A \otimes_{h} B$ is a primitive Banach algebra under the hypothesis that $A$ and $B$ are primitive $\mathrm{C}^{*}$-algebras. Furthermore, since $A \otimes_{h} B$ is a closed two-sided ideal of $\tilde{A} \otimes_{h} \tilde{B}$, it is enough to show that $\tilde{A} \otimes_{h} \tilde{B}$ is primitive. Thus we may assume, at the outset, that $A$ and $B$ are unital.

Let $M$ and $N$ be maximal left ideals of $A$ and $B$ respectively such that the largest two-sided ideal of $A$ (respectively, $B$ ) contained in $M$ (respectively $N$ ) is the zero ideal. Then the left ideal $M \otimes_{h} B+A \otimes_{h} N$ is closed in $A \otimes_{h} B$ [1;2.6]. Let $S$ be any left ideal of $A \otimes_{h} B$ containing both $M \otimes_{h} B+A \otimes_{h} N$ and a non-zero closed, two-sided ideal of $A \otimes_{h} B$. We shall show that $S=A \otimes_{h} B$.

Each non-zero closed two-sided ideal of $A \otimes_{h} B$ contains a non-zero simple tensor [1; 4.6], so there is a non-zero simple tensor $a \otimes b \in S$ such that the closed two-sided ideal $I$ generated by $a \otimes b$ is contained in $S$. On the other hand, since $A$ and $B$ are primitive, $I_{a} \nsubseteq M$ and $I_{b} \nsubseteq N$, where $I_{a}$ and $I_{b}$ are the smallest closed, two-sided ideals of $A$ and $B$ containing $a$ and $b$ respectively. Thus there exist $c, d \in A$ and $e, f \in B$ such that $c a d \notin M$ and $e b f \notin N$.

Since $M$ and $N$ are maximal left ideals in $A$ and $B$ respectively there exist $g \in A$ and $m \in M$ such that $g(c a d)+m=1_{A}$, and $h \in B$ and $n \in N$ such that $h(e b f)+n=1_{B}$. Hence

$$
\begin{aligned}
\left(1_{A} \otimes 1_{B}\right) & =(g c a d+m) \otimes(h e b f+n) \\
& =g c a d \otimes h e b f+g c a d \otimes n+m \otimes 1_{B} \\
& =(g c \otimes h e)(a \otimes b)(d \otimes f)+\left(1_{A} \otimes n-m \otimes n\right)+m \otimes 1_{B} \\
& \in I+(S-S)+S \subseteq S .
\end{aligned}
$$

Hence $S=A \otimes_{h} B$ as required. Thus if $T$ is a maximal left ideal of $A \otimes_{h} B$ containing $M \otimes_{h} B+A \otimes_{h} N$ then the only two sided-ideal of $A \otimes_{h} B$ contained in $T$ is the zero ideal. Thus $A \otimes_{h} B$ is primitive. Q.E.D.

Theorem 2.2 leaves open the interesting question whether the topologically irreducible *representation associated with the primitive ideal $P \otimes_{h} B+A \otimes_{h} Q$ is actually algebraically irreducible.

Corollary 2.3 Let $A$ and $B$ be separable $C^{*}$-algebras. Then

$$
\operatorname{Prim}_{*}\left(A \otimes_{h} B\right)=\operatorname{Prim}^{s}\left(A \otimes_{h} B\right)=\operatorname{Prim}\left(A \otimes_{h} B\right) .
$$


Proof. The equality of $\operatorname{Prim}^{s}\left(A \otimes_{h} B\right)$ and $\operatorname{Prim}_{*}\left(A \otimes_{h} B\right)$, for $A$ and $B$ separable, was established in [1; 5.15]. If $R \in \operatorname{Prim}\left(A \otimes_{h} B\right)$ then $R$ is, of course, prime and semisimple, so $R \in \operatorname{Prim}^{s}\left(A \otimes_{h} B\right)$ (without any separability hypothesis). Conversely, suppose that $R \in \operatorname{Prim}^{s}\left(A \otimes_{h} B\right)$. Then $R$ is closed and prime, so there exist closed, prime ideals $P \in I d(A)$ and $Q \in I d(B)$ such that $R=P \otimes_{h} B+A \otimes_{h} Q$ [1; 5.9(iii)]. But closed, prime ideals are primitive in separable $\mathrm{C}^{*}$-algebras, so $P \in \operatorname{Prim}(A)$ and $Q \in \operatorname{Prim}(B)$. Hence $R \in \operatorname{Prim}\left(A \otimes_{h} B\right)$ by Theorem 2.2. Q.E.D.

We now show that spectral synthesis is equivalent to the Hausdorffness of $\tau_{\infty}$ for $A \otimes_{h} B$. We use the embedding of $A \otimes_{h} B$ in $A \otimes_{\min } B$ mentioned earlier [5]. With an eye to subsequent work we make the following general definition.

Let $M$ be a Banach algebra, and suppose that there is a continuous norm $\gamma$ on $M$ such that $N$, the $\gamma$-completion of $M$, is a $\mathrm{C}^{*}$-algebra. In this paper $M$ will of course be $A \otimes_{h} B$ and $N$ will be $A \otimes_{\min } B$. Extending the earlier definition, we refer to those closed ideals in $M$ of the form $J \cap M(J \in I d(N))$ as upper ideals, and the set of upper ideals is denoted $I d^{u}(M)$. Note that if $I$ is an upper ideal of $M$ then in fact $I=J \cap M$ where $J$ is the closure of $I$ in $N$.

Definition 2.4 Let $M$ be a Banach algebra. We shall say that $M$ has property $(P)$ if $M$ satisfies the following conditions:

(a) there is a continuous norm $\gamma$ on $M$ such that $N$, the $\gamma$-completion of $M$, is a $\mathrm{C}^{*}$-algebra;

(b) every primitive ideal of $M$ is an upper ideal, i.e. $\operatorname{Prim}(M) \subseteq I d^{u}(M)$;

(c) there is a subset $R$ of $M \cap N_{s a}$ such that each $a \in R$ is contained in a completely regular, commutative Banach *-subalgebra $M_{a}$ of $M$ (where the norm and the involution on $M_{a}$ are those induced by $\left.N\right)$, and such that if $I \in I d^{u}(M)$ and $J \in I d(M)$ with $J \nsubseteq I$ then there exists $a \in R$ such that $M_{a} \cap J \nsubseteq I$.

Notice for later that if $M$ is a Banach algebra with property $(\mathrm{P})$ and $I$ is an upper ideal of $M$ then $M / I$ also has property $(\mathrm{P})$.

In Theorem 2.7 we show that the Haagerup tensor product of two $\mathrm{C}^{*}$-algebras has property $(\mathrm{P})$.

For the next lemma, recall Rickart's theorem, quoted before Theorem 1.8, which states that for any norm $\sigma$ on a completely regular, semisimple, commutative Banach algebra $A$, $\sigma(a) \geq \rho(a)$ for all $a \in A$, where $\rho$ denotes the uniform norm on $A$ [20].

Lemma 2.5 Suppose that $M$ is a Banach algebra with property $(P)$ and that a belongs to the subset $R$. Then

(i) if $J \in I d^{u}(M)$ then $M_{a} \cap J$ is a semisimple ideal in $M_{a}$,

(ii) $\left.\gamma\right|_{M_{a}}$ is the uniform norm on $M_{a}$.

Proof. (i) Let $K$ be a closed ideal of $N$ such that $J=K \cap M$. Then $M_{a} /\left(M_{a} \cap J\right)$ is isomorphic to a commutative * ${ }^{*}$ subalgebra, $C$ say, of $N / K$. The completion of $C$ in $N / K$ is an abelian $\mathrm{C}^{*}$-algebra, hence semisimple. Taking a completion reduces the spectral radius, so $C$ has no non-zero quasi-nilpotent elements. Thus $C$ is semisimple. 
(ii) Let $D \subseteq N$ be the $\gamma$-completion of $M_{a}$. Then $D$ is a commutative $\mathrm{C}^{*}$-subalgebra of $N$. Let $\Delta(D)$ be the character space of $D$. For $\phi \in \Delta(D)$, let $\tilde{\phi}$ denote the character obtained by restricting $\phi$ to $M_{a}$. Then for $c \in M_{a}$,

$$
\gamma(c)=\sup \{\phi(c): \phi \in \Delta(D)\}=\sup \{\tilde{\phi}(c): \phi \in \Delta(D)\} \leq \rho(c)
$$

where $\rho$ denotes the spectral radius on $M_{a}$. But $M_{a}$ is semisimple by part (i) (taking $J=\{0\})$, so the spectral radius $\rho$ is the uniform norm on $M_{a}$. Hence $\rho(c)=\gamma(c)$, by Rickart's theorem, since $\gamma$ is also a norm. Q.E.D.

Proposition 2.6 Let $M$ be a Banach algebra with property $(P)$ above. Then every upper ideal of $M$ is a $\tau_{\infty}$-closed point of $\operatorname{Id}(M)$. If $M$ has spectral synthesis then $\tau_{\infty}$ is Hausdorff on $\operatorname{Id}(M)$.

Proof. If $I$ is an upper ideal in $I d(M)$ then $M / I$ also has property (P), as we have observed, so we may assume that $I=\{0\}$. Next note that for any continuous norm $\rho$ on $M$ and for any $a \in R, \rho(a) \geq \gamma(a)$, by Rickart's theorem and Lemma 2.5(ii). Now suppose that $k \in \mathbf{N}$ and that $\left(\rho_{\alpha}\right)$ is a net of norms in $S_{k}$, converging to a seminorm $\rho \in S_{k}$. Let $a \in R$ and let $b \in M_{a} \cap \operatorname{ker} \rho$. Then $\rho_{\alpha}(b) \rightarrow 0$. Hence $\gamma(b)=0$, so $b=0$. Thus $\operatorname{ker} \rho=\{0\}$, by condition (c) of property (P), so $\rho$ is a norm on $M$. Hence $\{0\}$ is $\tau_{\infty}$-closed, as required.

Now suppose, additionally, that $M$ has spectral synthesis. Then every closed ideal of $M$ is semisimple, so condition (b) of property (P) implies that every closed ideal of $M$ is an upper ideal. Let $k \in \mathbf{N}$ and let $\left(\rho_{\alpha}\right)$ be an increasing net of seminorms in $S_{k}$ with limit $\rho$. By $[23 ; 2.2]$ we must show that $J:=\sup \operatorname{ker} \rho_{\alpha}$ coincides with $\operatorname{ker} \rho$. It is always the case that $J \subseteq \operatorname{ker} \rho$. Let $a \in R$ and let $b \in M_{a} \cap \operatorname{ker} \rho$. For each $\alpha$, let $\sigma_{\alpha}$ be the uniform norm on the algebra $M_{a} /\left(M_{a} \cap \operatorname{ker} \rho_{\alpha}\right)$, which is semisimple by Lemma 2.5(i), and let $\sigma$ be the uniform norm on the semisimple algebra $M_{a} /\left(M_{a} \cap J\right)$. Then by Rickart's theorem, $\rho_{\alpha}(b) \geq \sigma_{\alpha}(b) \geq \sigma(b)$ for each $\alpha$. Hence $\sigma(b)=0$, since $\rho_{\alpha}(b) \rightarrow 0$. Thus $b \in J$. It follows that $M_{a} \cap \operatorname{ker} \rho=M_{a} \cap J$, and hence that $J=\operatorname{ker} \rho$, by condition (c) of property (P). Q.E.D.

Theorem 2.7 Let $A$ and $B$ be $C^{*}$-algebras with Haagerup tensor product $A \otimes_{h} B$. Then every upper ideal is $\tau_{\infty}$-closed in $\operatorname{Id}\left(A \otimes_{h} B\right)$, and $A \otimes_{h} B$ has spectral synthesis if and only if $\tau_{\infty}$ is Hausdorff on $\operatorname{Id}\left(A \otimes_{h} B\right)$.

Proof. The fact that the Hausdorffness of $\tau_{\infty}$ implies spectral synthesis follows from Theorem 1.11 and the fact, already mentioned, that weak spectral synthesis and spectral synthesis are equivalent for $A \otimes_{h} B$. The rest of the theorem will follow from Proposition 2.6 once we have established that $A \otimes_{h} B$ has property (P) of Definition 2.4.

We have already observed that conditions (a) and (b) of Property (P) are satisfied. Now let $R$ be the set $\left\{a \otimes b: a \in A^{+}, b \in B^{+}\right\}$. Then for $a \otimes b \in R$ the commutative subalgebra $C^{*}(a) \otimes_{h} C^{*}(b) \subseteq A \otimes_{h} B$ is a completely regular Banach *-algebra. Let $I \in I d^{u}\left(A \otimes_{h} B\right)$ and let $J \in I d\left(A \otimes_{h} B\right)$ with $J \nsubseteq I$. Then $J_{l} \nsubseteq I$ since $J \nsubseteq I$. But $J_{l}$ is generated by the product ideals which it contains $[1 ; 6.7(\mathrm{i})]$, and each product ideal is generated by its positive simple tensors, so there exists a simple tensor $a \otimes b \in J_{l} \backslash I$ with 
$a \in A^{+}$and $b \in B^{+}$. This establishes condition (c) of property (P). Hence $A \otimes_{h} B$ has property $(\mathrm{P})$, as required. Q.E.D.

\section{The topology $\tau_{r}$ on $\operatorname{Id}\left(A \otimes_{h} B\right)$}

In this section we consider the topology $\tau_{r}$ on $\operatorname{Id}\left(A \otimes_{h} B\right)$, showing that if $\operatorname{Prime}(A)$ and Prime $(B)$ are compact $\mathrm{T}_{1}$-spaces, then $A \otimes_{h} B$ has spectral synthesis if and only if $\tau_{r}$ is Hausdorff on $\operatorname{Id}\left(A \otimes_{h} B\right)$. The proof uses the theory of continuous lattices and bitopological spaces.

We begin by looking at the lattice $I d^{u}\left(A \otimes_{h} B\right)$ of upper ideals. Recall that these are precisely the semisimple ideals of $A \otimes_{h} B$. Thus they are in bijective correspondence with the open (or closed) subsets of $\operatorname{Prim}\left(A \otimes_{h} B\right)$, using the hull-kernel process.

We need to recall the definition of a continuous lattice. Let $L$ be a complete lattice and let $x, y \in L$. Then $x$ is way-below $y$, written $x \ll y$, if whenever $S$ is a subset of $L$ with $y \leq \sup S$ there is a finite subset $F$ of $S$ such that $x \leq \sup F$, see [12]. If for each $y \in L, y=\sup \{x \in L: x \ll y\}$ then $L$ is called a continuous lattice. A continuous lattice carries three important topologies, as follows. The lower topology on $L$ is the topology generated by the sets $\{x \in L: x \nsupseteq y\}$ as $y$ varies through $L$ (in exact analogy with the lower topology on $\operatorname{Id}(A)$ defined in the Introduction). A base for the Scott topology on $L$ is given by sets of the form $\{y \in L: y \gg x\}$ as $x$ varies through $L$. The Lawson topology on $L$ is the join of the Scott and lower topologies. The Lawson topology on a continuous lattice is compact and Hausdorff [12; III.1.10].

An element $p$ in a lattice $L$ is said to be prime if whenever $x, y \in L$ with $x \wedge y \leq p$ then either $x \leq p$ or $y \leq p$. Let Prime $(L)$ denote the set of proper prime elements of $L$ (in lattice theory it is conventional to include the 'top' as well, but we are excluding it, in harmony with the convention in ring theory). As in the earlier setting, the lower topology coincides with the hull-kernel topology on Prime $(L)$. A subset $S$ of Prime $(L)$ is said to be saturated if $x \in S$ and $y \in \operatorname{Prime}(L)$ with $y \leq x$ implies that $y \in S$. We shall use the fact that a subset $S$ of Prime $(L)$ is open in the relative Scott topology if and only if Prime $(L) \backslash S$ is saturated and hull-kernel compact [12; V.5.1]. For $x \in L$, let $\operatorname{hull}(x)=\{p \in \operatorname{Prime}(L): p \geq x\}$. The spectral theorem for a continuous, distributive lattice $L$ is that the correspondence $x \longleftrightarrow h u l l(x)$ is a lattice isomorphism between $L$ and the lattice of closed subsets of Prime $(L)$ in the lower topology [12; V.5,5]. In particular, $x=\bigwedge \operatorname{hull}(x)$ for each element $x$ in a continuous, distributive lattice. We shall also use the fact that if $x$ and $y$ are elements of a continuous distributive lattice $L$ then $x \ll y$ if and only if there exists a Scott open set $M$ such that $h u l l(x) \supseteq M \supseteq \operatorname{hull}(y)$, see [12; V.5.6(ii)]. The following result is standard, but it seems best to include the short proof.

Lemma 3.1 Let $L$ be a continuous distributive lattice. Let $x \in L$ and let $\left(x_{\alpha}\right)$ be a net in $L$ converging to $x$ in the Scott topology. Let $V$ be a Scott open subset of Prime $(L)$ containing hull $(x)$. Then eventually hull $\left(x_{\alpha}\right) \subseteq V$.

Proof. Suppose to the contrary. Then because $V^{c}:=\operatorname{Prime}(L) \backslash V$ is hull-kernel compact, we may suppose, by passing to a subnet if necessary, that there exists $p \in V^{c}$ and a net 
$\left(p_{\alpha}\right)$ with $p_{\alpha} \in V^{c} \cap \operatorname{hull}\left(x_{\alpha}\right)$ for each $\alpha$, such that $p_{\alpha} \rightarrow p$ as $\alpha \rightarrow \infty$, in the hull-kernel topology. Since $L$ is a continuous lattice, there exists $y \in L$ with $y \ll x$ and $y \not \leq p$. The set $\{z \in L: z \gg y\}$ is a Scott-open neighbourhood of $x$, so eventually $x_{\alpha} \gg y$. Hence eventually $x_{\alpha} \geq y$, so eventually $\operatorname{hull}\left(x_{\alpha}\right)$ is contained in the set $h u l l(y)$ which is closed in the hull-kernel topology. Since $p \notin h u l l(y)$, we have a contradiction. Q.E.D.

We observed near the beginning of Section 2 that $\operatorname{Prim}^{s}\left(A \otimes_{h} B\right)=\operatorname{Prime}\left(A \otimes_{h} B\right)$ is homeomorphic to $\operatorname{Prime}(A) \times \operatorname{Prime}(B)$, and is therefore locally compact. On the other hand, the lattice $I d^{u}\left(A \otimes_{h} B\right)$ is isomorphic to the lattice of open subset of $\operatorname{Prim}(A)$, which in turn is isomorphic to the lattice of open subsets of $\operatorname{Prim}^{s}\left(A \otimes_{h} B\right)$. It follows from [12; V.5.10], therefore, that $I d^{u}\left(A \otimes_{h} B\right)$ is a continuous distributive lattice. Furthermore, $\operatorname{Prime}\left(I d^{u}\left(A \otimes_{h} B\right)\right)$ is precisely $\operatorname{Prime}\left(A \otimes_{h} B\right)[1 ; 5.9]$. The next proposition describes the lower, Scott, and Lawson topologies on $I d^{u}\left(A \otimes_{h} B\right)$. For $I \in I d^{u}\left(A \otimes_{h} B\right)$ and $x \in A \otimes_{h} B$, define

$$
\|x+I\|^{u}=\sup \{\|x+P\|: P \in \operatorname{hull}(I)\},
$$

where $\operatorname{hull}(I)=\left\{P \in \operatorname{Prime}\left(A \otimes_{h} B\right): P \supseteq I\right\}$. We shall refer to the functions $I \mapsto$ $\|x+I\|^{u}\left(I \in I d^{u}\left(A \otimes_{h} B\right), x \in A \otimes_{h} B\right)$ as upper-norm functions. Note that for $P \in \operatorname{Prime}\left(A \otimes_{h} B\right),\|x+P\|=\|x+P\|^{u}\left(x \in A \otimes_{h} B\right)$. It was shown in $[2 ; 3.4]$ that for $x \in A \otimes_{h} B$, the function $P \mapsto\|x+P\|\left(P \in \operatorname{Prime}\left(A \otimes_{h} B\right)\right)$ is lower semi-continuous on $\operatorname{Prime}\left(A \otimes_{h} B\right)$.

In Proposition 3.2 and Theorem 3.6 we shall use the hypothesis that $\operatorname{Prime}(A)$ and Prime $(B)$ are compact, for the $\mathrm{C}^{*}$-algebras $A$ and $B$. Note that for a $\mathrm{C}^{*}$-algebra $A$, $\operatorname{Prime}(A)$ is compact if and only if $\operatorname{Prim}(A)$ is compact. This is easily seen from the 'open-cover' version of compactness, using the natural extension of a cover of $\operatorname{Prim}(A)$ to a cover of $\operatorname{Prime}(A)$ by saturation, together with the fact that every proper closed prime ideal of $A$ is contained in a primitive ideal of $A$. One circumstance in which $\operatorname{Prim}(A)$ is compact is, of course, when $A$ is unital.

Proposition 3.2 Let $A$ and $B$ be $C^{*}$-algebras with Prime $(A)$ and Prime $(B)$ compact in the hull-kernel topology. Let $C=A \otimes_{h} B$ be the Haagerup tensor product of $A$ and $B$ and let $I d^{u}(C)$ be the lattice of upper ideals of $C$. Then the lower, Scott, and Lawson topologies on $I d^{u}(C)$ are the weakest with respect to which the upper-norm functions $I \mapsto\|x+I\|^{u}$, $\left(x \in C, I \in I d^{u}(C)\right)$, are respectively lower semi-continuous, upper semi-continuous, and continuous.

Proof. As temporary notation, let $\tau_{l c}, \tau_{u c}$, and $\tau_{c}$ be the weakest topologies on $I d^{u}(C)$ with respect to which the upper-norm functions are respectively lower semi-continuous, upper semi-continuous, and continuous. First we show that $\tau_{l c}$ coincides with the lower topology.

For each $x \in C$, the set $\left\{I \in I d^{u}(C):\|x+I\|^{u}>0\right\}$ is $\tau_{l c^{-}}$-open. Since these sets give a sub-base for the lower topology, it is immediate that $\tau_{l c}$ is stronger than the lower topology. Now let $\left(I_{\alpha}\right)$ be a net in $I d^{u}(C)$ converging in the lower topology to some $I \in I d^{u}(C)$, and suppose, without loss of generality, that $x \in C$ with $\|x+I\|^{u}=1$. Let $1>\epsilon>0$ be given. Then there exists $P \in \operatorname{Prim}(C)$ with $P \supseteq I$ such that $\|x+P\|>1-\frac{\epsilon}{2}$. By the lower 
semicontinuity of the upper-norm functions on $\operatorname{Prim}(C)[2 ; 3.4]$, there is a neighbourhood $X$ of $P$ in $\operatorname{Prim}(C)$ such that $\|x+Q\|>1-\epsilon$ for all $Q \in X$. Let $y \in C \backslash P$ be such that the open subset $Y=\{Q \in \operatorname{Prim}(C): y \notin Q\}$ of $\operatorname{Prim}(C)$ is a neighbourhood of $P$ contained in $X$. Then $y \notin I$, so eventually $y \notin I_{\alpha}$. Thus eventually there is, for each $\alpha$, a primitive ideal $P_{\alpha}$ of $C$ containing $I_{\alpha}$ such that $y \notin P_{\alpha}$. Hence $P_{\alpha} \in Y \subseteq X$, so $\left\|x+P_{\alpha}\right\|>1-\epsilon$. Thus eventually $\left\|x+I_{\alpha}\right\|^{u}>1-\epsilon$. This shows that the lower topology is stronger than $\tau_{l c}$. Hence $\tau_{l c}$ is the lower topology on $I d^{u}(C)$.

Next we show that $\tau_{u c}$ is weaker than the Scott topology on $I d^{u}(C)$. We begin by showing that the relative $\tau_{u c}$ topology is weaker than the relative Scott topology on Prime $(C)$. Recall that Prime $(C)$ is in bijective correspondence with $\operatorname{Prime}(A) \times \operatorname{Prime}(B)$, the correspondence being given by

$$
P \otimes_{h} B+A \otimes_{h} B \longleftrightarrow(P, Q) \in \operatorname{Prime}(A) \times \operatorname{Prime}(B) .
$$

Recall too that the usual $\mathrm{C}^{*}$-norm functions are upper semi-continuous on $\operatorname{Prime}(A)$ and Prime $(B)$ in the relative Scott topologies $[14 ; 7.2(\mathrm{~b})]$. Thus it follows from the remark after $[2 ; 3.1]$ that the norm functions $I \mapsto\|x+P\|,(x \in C, P \in \operatorname{Prime}(C))$, are upper semi-continuous on $\operatorname{Prime}(C)$ when $\operatorname{Prime}(C)$ is equipped with the product Scott topology from Prime $(A) \times \operatorname{Prime}(B)$. We need only show, therefore, that the product Scott topology is weaker than the Scott topology on Prime $(C)$. This follows from the fact that if $X$ and $Y$ are compact, saturated subsets of $\operatorname{Prime}(A)$ and $\operatorname{Prime}(B)$ respectively then

$$
Z:=\left\{P \otimes_{h} B+A \otimes_{h} Q:(P, Q) \in(X \times \operatorname{Prime}(B)) \cup(\operatorname{Prime}(A) \times Y)\right\}
$$

is a compact saturated subset of Prime $(C)$. Hence the set

$$
\operatorname{Prime}(C) \backslash Z \cong(\operatorname{Prime}(A) \backslash X) \times(\operatorname{Prime}(B) \backslash Y)
$$

is open in Prime $(C)$ in the Scott topology. Since sets of the form $(\operatorname{Prime}(A) \backslash X) \times$ (Prime $(B) \backslash Y)$ form a base for the product Scott topology, it follows that the product Scott topology on Prime $(C)$ is weaker than the Scott topology. Hence the relative $\tau_{u c}$ topology is weaker than the relative Scott topology on Prime $(C)$.

Now we extend this to the whole of $I d^{u}(C)$. Let $I \in I d^{u}(C)$ and let $\left(I_{\alpha}\right)$ be a net in $I d^{u}(C)$ converging to $I$ in the Scott topology. Let $x \in C$ and let $\epsilon>0$ be given. Then by the previous paragraph, for each $P \in \operatorname{hull}(I)$ there is a subset $V_{P}$ of $\operatorname{Prime}(C)$ which is open in the relative Scott topology and such that $\|x+Q\|<\|x+P\|+\epsilon$ for all $Q \in V_{P}$. Set $V=\bigcup\left\{V_{P}: P \in \operatorname{hull}(I)\right\}$. Then $V$ is a Scott open subset of Prime $(C)$ containing $h u l l(I)$. Thus by Lemma 3.1, eventually $h u l l\left(I_{\alpha}\right) \subseteq V$, implying that eventually $\left\|x+I_{\alpha}\right\|^{u}<\|x+I\|^{u}+\epsilon$. Thus $\tau_{u c}$ is weaker than the Scott topology on $I d^{u}(C)$.

To show that $\tau_{u c}$ is stronger than the Scott topology on $I d^{u}(C)$, let $I, J \in I d^{u}(C)$ with $I \nsubseteq J$. Let $x \in I \backslash J$, with $\|x+J\|^{u}=1$. Then the sets

$$
\left\{K \in I d^{u}(C):\|x+K\|^{u}>1 / 2\right\}
$$

and

$$
\left\{K \in I d^{u}(C):\|x+K\|^{u}<1 / 2\right\}
$$


are disjoint neighbourhoods of $J$ and $I$, open in the lower and $\tau_{u c}$ topologies respectively. Thus the bitopological space $\left(I d^{u}(C)\right.$, lower, $\left.\tau_{u c}\right)$ is 'pseudo-Hausdorff' in the sense of [17]. On the other hand, the bitopological space $\left(I d^{u}(C)\right.$, lower, Scott) is 'stable', being 'joincompact' (the definitions of these various properties are given in $[17]$ ), so $[17 ; 3.4(\mathrm{~d})]$ shows that $\tau_{u c}$ is stronger than the Scott topology. Thus $\tau_{u c}$ and the Scott topology coincide.

Finally, since $\tau_{c}$ is the join of the $\tau_{l c}$ and $\tau_{u c}$ topologies, while the Lawson topology is the join of the lower and Scott topologies, it follows immediately that $\tau_{c}$ coincides with the Lawson topology. Q.E.D.

Lemma 3.3 Let $A$ and $B$ be $C^{*}$-algebras with Haagerup tensor product $A \otimes_{h} B$. Let $I, J \in I d\left(A \otimes_{h} B\right)$ with $J^{u} \ll I^{u}$ in $I d^{u}\left(A \otimes_{h} B\right)$. Then $J \subseteq I_{l}$.

Proof. Let $\left(I_{\alpha}\right)$ be the net of finite sums of product ideals contained in $I_{l}$, upward directed by inclusion. Then $\left(I_{\alpha}^{u}\right)$ is a directed set in $I d^{u}\left(A \otimes_{h} B\right)$. If $P$ is any primitive ideal of $A \otimes_{h} B$ not containing $I$, then there is a product ideal contained in $I$ but not in $P$ (see the proof of Theorem 2.7). It follows that $I_{\alpha}^{u} \nearrow I^{u}$ in the lattice $I d^{u}\left(A \otimes_{h} B\right)$. Thus eventually $I_{\alpha}^{u} \supseteq J^{u} \supseteq J$, by assumption. But each $I_{\alpha}$ has spectral synthesis [1; 6.4], so $I_{\alpha}=I_{\alpha}^{u}$. Thus eventually $I_{\alpha} \supseteq J$. Since $I_{l}$ is the norm closure of the product ideals which it contains $[1 ; 6.7(\mathrm{i})]$, we have shown that $I_{l} \supseteq J$. Q.E.D.

We now need some information about quotient norms.

Lemma 3.4 Let $A$ and $B$ be $C^{*}$-algebras. Let $\left\{I_{i}\right\}$ and $\left\{J_{i}\right\}(1 \leq i \leq n)$ be finite sets of closed ideals of $A$ and $B$ respectively such that all the sets $\left\{P \in \operatorname{Prim}(A): P \supseteq I_{i}\right\}$ $(1 \leq i \leq n)$ are disjoint in $\operatorname{Prim}(A)$ and all the sets $\left\{Q \in \operatorname{Prim}(B): Q \supseteq J_{i}\right\}(1 \leq i \leq n)$ are disjoint in $\operatorname{Prim}(B)$. Set $K=\bigcap_{i=1}^{n}\left(I_{i} \otimes_{h} B+A \otimes_{h} J_{i}\right)$. Then for $x \in A \otimes_{h} B$,

$$
\|x+K\|=\max \left\{\left\|x+\left(I_{i} \otimes_{h} B+A \otimes_{h} J_{i}\right)\right\|: 1 \leq i \leq n\right\} .
$$

Proof. Set $G=\bigcap_{i=1}^{n} I_{i}$ and $H=\bigcap_{i=1}^{n} J_{i}$. Then $A \otimes_{h} B /\left(G \otimes_{h} B+A \otimes_{h} H\right)$ is isometrically isomorphic to $A / G \otimes_{h} B / H$, see $[1 ; 2.6]$. But $A / G \cong \bigoplus_{i=1}^{n} A / I_{i}$ and $B / H \cong \bigoplus_{i=1}^{n} B / J_{i}$, so $A / G \otimes_{h} B / H$ is isometrically isomorphic to $\bigoplus_{i, j=1}^{n} A / I_{i} \otimes_{h} B / J_{j}$. Hence

$$
\left\|x+\left(G \otimes_{h} B+A \otimes_{h} H\right)\right\|=\max \left\{\left\|x+\left(I_{i} \otimes_{h} B+A \otimes_{h} J_{j}\right)\right\|: 1 \leq i, j \leq n\right\} .
$$

Thus $A \otimes_{h} B / K$ is isometrically isomorphic to $\bigoplus_{i=1}^{n} A / I_{i} \otimes_{h} B / J_{i}$ and

$$
\|x+K\|=\max \left\{\left\|x+\left(I_{i} \otimes_{h} B+A \otimes_{h} J_{i}\right)\right\|: 1 \leq i \leq n\right\} .
$$

Q.E.D.

In fact we only use Lemma 3.4 in the case when the ideals $I_{i}$ and $J_{i}$ are maximal ideals of $A$ and $B$ respectively. We state this case separately.

Proposition 3.5 Let $A$ and $B$ be $C^{*}$-algebras and let $F=\left\{J_{1}, \ldots, J_{n}\right\}$ be a finite set of maximal ideals of $A \otimes_{h} B$. Set $I(F)=\bigcap_{i=1}^{n} J_{i}$. Then for each $x \in A \otimes_{h} B$,

$$
\|x+I(F)\|=\max \left\{\left\|x+J_{i}\right\|: 1 \leq i \leq n\right\} .
$$


Proof. For each $J_{i} \in F$, there exist maximal ideals $M_{i}$ of $A$ and $N_{i}$ of $B$ such that $J_{i}=M_{i} \otimes_{h} B+A \otimes_{h} N_{i}[1 ; 5.6]$. The sets $\left\{P \in \operatorname{Prim}(A): P \supseteq M_{i}\right\}(1 \leq i \leq n)$ and $\left\{Q \in \operatorname{Prim}(B): Q \supseteq N_{i}\right\}(1 \leq i \leq n)$ are trivially disjoint in $\operatorname{Prim}(A)$ and $\operatorname{Prim}(B)$ respectively. Hence the result follows from Lemma 3.4. Q.E.D.

In the case when $A$ and $B$ are commutative $\mathrm{C}^{*}$-algebras, Proposition 3.5 shows that every finite subset of $\operatorname{Max}\left(A \otimes_{h} B\right)$ is a Helson set with Helson constant 1 . Thus $A \otimes_{h} B$ has the Helson property with constant 1 , so it follows from Theorem 1.9 that $A \otimes_{h} B$ has spectral synthesis if and only if $\tau_{r}$ is Hausdorff. Our final result, the main one of this section, is a partial extension of this to the non-commutative situation. The proof is similar to that of Theorem 1.9 but with a few extra turns.

Theorem 3.6 Let $A$ and $B$ be $C^{*}$-algebras with Prime $(A)$ and Prime $(B)$ compact $T_{1}$ spaces. Then the following are equivalent:

(i) $A \otimes_{h} B$ has spectral synthesis,

(ii) $\operatorname{Id}\left(A \otimes_{h} B\right)$ is $\tau_{r}$-Hausdorff.

Proof. (i) $\Rightarrow$ (ii) follows from Theorem 2.7 and $[24 ; 3.1 .1]$.

(ii) $\Rightarrow$ (i). Suppose that spectral synthesis fails. We shall show that $\tau_{r}$ is not Hausdorff on $\operatorname{Id}\left(A \otimes_{h} B\right)$. The hypotheses on $\operatorname{Prime}(A)$ and $\operatorname{Prime}(B)$ imply that $\operatorname{Prime}\left(A \otimes_{h} B\right)=$ $\operatorname{Max}\left(A \otimes_{h} B\right)$. Let $I \in I d\left(A \otimes_{h} B\right)$ with $I_{l} \neq I^{u}$. Let $N=\operatorname{hull}\left(I^{u}\right)$, and let $\left(N_{\alpha}^{\prime}\right)_{\alpha}$ be the net of decreasing, Scott open subsets of $\operatorname{Max}\left(A \otimes_{h} B\right)$ containing $N$. Let $\left(N_{\alpha}\right)_{\alpha}$ be the net of decreasing, hull-kernel closed subsets of $\operatorname{Max}\left(A \otimes_{h} B\right)$, where for each $\alpha, N_{\alpha}$ is the hullkernel closure of $N_{\alpha}^{\prime}$. Since $\operatorname{Max}\left(A \otimes_{h} B\right)$ is locally compact in the hull-kernel topology, and a subset of $\operatorname{Max}\left(A \otimes_{h} B\right)$ is open in the Scott topology if and only its complement is compact and saturated, it follows that $\bigcap_{\alpha} N_{\alpha}=N$.

For a hull-kernel closed subset $M$ of $\operatorname{Max}\left(A \otimes_{h} B\right)$, let $I(M)=\bigcap\{Q: Q \in M\}$. Then $\left(I\left(N_{\alpha}\right)\right)_{\alpha}$ is an increasing net in $I d^{u}\left(A \otimes_{h} B\right)$, and each $I\left(N_{\alpha}\right) \ll I^{u}$ by the remark before Lemma 3.1. Hence $I\left(N_{\alpha}\right) \subseteq I_{l}$ for each $\alpha$, by Lemma 3.3. Thus $J:=\overline{\bigcup_{\alpha} I\left(N_{\alpha}\right)} \subseteq I_{l}$. On the other hand, $I_{l}$ is the smallest closed ideal with hull equal to $N[1 ; 6.6(\mathrm{iv})]$, so $J=I_{l}$. For each $\alpha$, let $\left(F_{\beta(\alpha)}\right)_{\beta(\alpha)}$ be the increasing net of finite subsets of $N_{\alpha}$. Then $\left(I\left(F_{\beta(\alpha)}\right)\right)_{\beta(\alpha)}$ is a decreasing net in $\operatorname{Id}\left(A \otimes_{h} B\right)$, and $\bigcap_{\beta(\alpha)} I\left(F_{\beta(\alpha)}\right)=I\left(N_{\alpha}\right)$. Hence

$$
I\left(F_{\beta(\alpha)}\right) \stackrel{\beta(\alpha)}{\longrightarrow} I\left(N_{\alpha}\right) \quad\left(\tau_{r}\right)
$$

in $I d\left(A \otimes_{h} B\right)$ by Lemma 0.1. But $I\left(N_{\alpha}\right) \rightarrow I_{l}\left(\tau_{r}\right)$, also by Lemma 0.1 , so if $\left(I\left(F_{\gamma}\right)\right)_{\gamma}$ denotes the 'diagonal' net, see $\left[16 ; \S 2\right.$, Theorem 4], then $I\left(F_{\gamma}\right) \rightarrow I_{l}\left(\tau_{r}\right)$ in $\operatorname{Id}\left(A \otimes_{h} B\right)$. Now suppose that $x \notin I^{u}$. Then there exists $P \in \operatorname{hull}(I)$ such that $x \notin P$. For each $\alpha$, eventually $P \in F_{\beta(\alpha)}$, so eventually

$$
\left\|x+I\left(F_{\beta(\alpha)}\right)\right\| \geq\|x+P\|>0 .
$$

Hence the 'diagonal' net $I\left(F_{\gamma}\right) \rightarrow I^{u}\left(\tau_{n}\right)$. On the other hand, if $x \in I^{u}$ and $\epsilon>0$ then by Proposition 3.2 there exists a Scott open subset $M$ of $\operatorname{Prim}\left(A \otimes_{h} B\right)=\operatorname{Max}\left(A \otimes_{h} B\right)$ 
containing $N$ such that $\|x+Q\|<\epsilon$ for all $Q \in M$. Since $\operatorname{Max}\left(A \otimes_{h} B\right) \backslash M$ is compact and $\cap N_{\alpha}=N$, there exists $\alpha_{0}$ such that for $\alpha \geq \alpha_{0}, N_{\alpha} \subseteq M$. Hence for $\alpha \geq \alpha_{0}$,

$$
\left\|x+I\left(F_{\beta(\alpha)}\right)\right\|<\epsilon
$$

for all $\beta(\alpha)$, by Proposition 3.5. Hence $I\left(F_{\gamma}\right) \rightarrow I^{u}\left(\tau_{u}\right)$, using $[24 ; 2.1]$, so

$$
I\left(F_{\gamma}\right) \rightarrow I^{u}\left(\tau_{r}\right)
$$

Since $I_{l} \neq I^{u}, \tau_{r}$ is not Hausdorff. Q.E.D.

Theorem 3.6 is sufficiently general to make one think that the result probably holds true for all $\mathrm{C}^{*}$-algebras $A$ and $B$.

\section{References}

[1] ALLEN, S.D.; SINCLAIR, A.M.; SMITH, R.R., The ideal structure of the Haagerup tensor product of $\mathrm{C}^{*}$-algebras, J. reine angew. Math., 442 (1993) 111-148.

[2] ARCHBOLD, R.J.; KANIUTH, E.; SCHLICHTING, G.; SOMERSET, D.W.B., Ideal spaces of the Haagerup tensor product of $\mathrm{C}^{*}$-algebras, Internat. J. Math., 8 (1997) $1-29$.

[3] BECKHOFF, F., Topologies on the space of ideals of a Banach algebra, Stud. Math., 115 (1995) 189-205.

[4] BECKHOFF, F., Topologies on the ideal space of a Banach algebra and spectral synthesis, Proc. Amer. Math. Soc., 125 (1997) 2859-2866.

[5] BLECHER, D.P., Geometry of the tensor product of $\mathrm{C}^{*}$-algebras, Math. Proc. Camb. Phil. Soc., 104 (1988) 119-127.

[6] BLECHER, D.P., Tensor products which do not preserve operator algebras, Math. Proc. Camb. Phil. Soc., 108 (1990) 395-403.

[7] BOHNENBLUST, H.F.; KARLIN, S., Geometrical properties of the unit sphere of Banach algebras, Ann. Math., (2) 62 (1955) 217-229.

[8] BONSALL, F.F.; DUNCAN, J., Complete Normed Algebras, Springer-Verlag, New York, 1973.

[9] COHN, P.M., Algebra, Vol. 3 (2nd edn.), Wiley, Chichester, 1991.

[10] FEINSTEIN, J.F.; SOMERSET, D.W.B., A note on ideal spaces of Banach algebras, Bull. London Math. Soc., 30 (1998) 611-617.

[11] J.F. FEINSTEIN, D.W.B. SOMERSET, Strong regularity for uniform algebras, in Contemporary Math., 232, 'Proceedings of the 3rd Function Spaces Conference, Edwardsville, Illinois, May 1998', ed. K. Jarosz, pp. 139-149, Amer. Math. Soc., Rhode Island, 1999.

[12] GIERZ, G.; HOFMANN, K.H.; KEIMEL, K.; LAWSON, J.; MISLOVE, M.; SCOTT, D.S., A Compendium of Continuous Lattices, Springer-Verlag, New York, 1980.

[13] GRAHAM, C.C.; McGEHEE, O.C., Essays in Commutative Harmonic Analysis, Springer-Verlag, New York, 1979. 
[14] HENRIKSEN, M.; KOPPERMAN, R.; MACK, J.; SOMERSET, D.W.B., Joincompact spaces, continuous lattices, and $\mathrm{C}^{*}$-algebras, Algebra Universalis, 38 (1997) 289323.

[15] KAPLANSKY, I., The structure of certain operator algebras, Trans. Amer. Math. Soc., 70 (1951) 219-255.

[16] KELLEY, J.L., General Topology, Van Nostrand, Princeton, 1955.

[17] KOPPERMAN, R., Asymmetry and duality in topology, Gen. Top. and Appl., 66 (1995) 1-39.

[18] PALMER, T.W., Banach Algebras and the General Theory of *-Algebras, Vol. 1, C.U.P., New York, 1994.

[19] PAULSEN, V.I.; SMITH, R.R., Multilinear maps and tensor norms on operator systems, J. Funct. Anal. 73 (1987) 258-276.

[20] RICKART, C.E., On spectral permanence for certain Banach algebras, Proc. Amer. Math. Soc., 4 (1953) 191-196.

[21] RICKART, C.E., General Theory of Banach Algebras, Van Nostrand, London, 1960.

[22] RUDIN, W, Fourier Analysis on Groups, Interscience, New York, 1962.

[23] SOMERSET, D.W.B., Spectral synthesis for Banach algebras, Quart. J. Math. Oxford, (2) 49 (1998) 501-521.

[24] SOMERSET, D.W.B., Ideal spaces of Banach algebras, Proc. London Math. Soc., (3) 78 (1999) 369-400.

[25] ZELAZKO, W., Banach Algebras, Elsevier, Warsaw, 1973.

School of Mathematical Sciences

University of Nottingham

NG7 2RD

U.K.

email: Joel.Feinstein@nottingham.ac.uk

Department of Mathematical Sciences

University of Aberdeen

AB24 3UE

U.K.

e-mail: ds@maths.abdn.ac.uk 